\title{
Hyperbolic Maxwell variational inequalities of the second kind
}

Yousept, Irwin

This text is provided by DuEPublico, the central repository of the University Duisburg-Essen.

This version of the e-publication may differ from a potential published print or online version.

URN: urn:nbn:de:hbz:464-20180910-105702-7

Link: https://duepublico.uni-duisburg-essen.de:443/servlets/DocumentServlet?id=46817

Source: Deutsche Forschungsgemeinschaft (DFG), Priority Programme 1962: SPP 1962;

Preprint Number SPP1962-069 https://spp1962.wias-berlin.de/preprints.php 


\title{
HYPERBOLIC MAXWELL VARIATIONAL INEQUALITIES OF THE SECOND KIND
}

\author{
IRWIN YOUSEPT *
}

\begin{abstract}
In this note, we provide an existence and uniqueness theorem for the weak and strong solutions to a general class of hyperbolic Maxwell variational inequalities of the second kind. The tools for our analysis are the semigroup theory for Maxwell's equations, the Moreau-Yosida regularization theory and the subdifferential analysis.
\end{abstract}

Key words. hyperbolic Maxwell variational inequalities of the second kind, well-posedness.

AMS subject classifications. 35Q60, 35L85.

1. Introduction. Physical phenomena in electromagnetism can lead to hyperbolic variational inequalities with a Maxwell structure. They include application problems arising from electromagnetic processes in polarizable media and Bean's critical-state model in high-temperature superconductivity. The very first study on hyperbolic variational inequalities in electromagnetism goes back to Duvaut and Lions [9]. By employing the Galerkin-ansatz and a parabolic regularization technique, they [9, Section 8] proved an existence and uniqueness result for the strong solution to a hyperbolic obstacle-type Maxwell variational inequality (VI of the first kind). Some years later, Milani $[14,15]$ extended their results to the case of time-dependent obstacle set. Quite recently, the author [24] shown that the Bean critical-state model for high-temperature superconductors [8] leads to a hyperbolic Maxwell variational inequality of the second kind. The optimal control problem of this variational inequality was analyzed in [25] (cf. also [23]). We note that the analysis of the Bean criticalstate model governed by the eddy current equations goes back to Prigozhin [19-21] and Barrett and Prigozhin [4-7]. The corresponding model governed by the Maxwell equations was considered in $[11,12]$. We also refer the reader to $[2,16,17]$ for parabolic and elliptic variational inequalities of a p-curl type arising from the power law for the eddy current equations.

This note analyzes a general class of hyperbolic Maxwell variational inequalities of the second kind on an open set $\Omega \subseteq \mathbb{R}^{3}$ representing the physical medium, where electromagnetic fields are acting. Our main result is the existence and uniqueness theorem for the strong and weak solutions. The main tools for our analysis are the semigroup theory for Maxwell's equations, the Moreau-Yosida approximation theory and the set-valued subdifferential analysis. Our paper is organized as follows. In the upcoming section, we introduce all the function spaces used in our analysis, including the definition of the Maxwell operator. Some well-known properties of the Maxwell operator are also mentioned in this section. In Section 3, we present our main existence and uniqueness theorem. The proof is presented in Sections 4 and 5.

2. Preliminaries. We introduce the Hilbert space

$$
\boldsymbol{H}(\operatorname{curl}):=\left\{\boldsymbol{q} \in \boldsymbol{L}^{2}(\Omega) \mid \operatorname{curl} \boldsymbol{q} \in \boldsymbol{L}^{2}(\Omega)\right\},
$$

\footnotetext{
*Universität Duisburg-Essen, Fakultät für Mathematik, Thea-Leymann-Str. 9, D-45127 Essen, Germany, irwin.yousept@uni-due.de. This work was supported by the German Research Foundation Priority Programm DFG SPP 1962 "Non-smooth and Complementarity-based Distributed Parameter Systems: Simulation and Hierarchical Optimization", Project YO 159/2-1.
} 
where the curl-operator is understood in the sense of distributions. Note that, for a given Hilbert space $V$, we use the notation $\|\cdot\|_{V}$ and $(\cdot, \cdot)_{V}$ for a standard norm and a standard scalar product in $V$. A bold typeface is used to indicate a three-dimensional vector function or a Hilbert space of three-dimensional vector functions. As usual, $\mathcal{C}_{0}^{\infty}(\Omega)$ stands for the space of all infinitely differentiable three-dimensional vector functions with compact support contained in $\Omega$. We denote the closure of $\mathcal{C}_{0}^{\infty}(\Omega)$ with respect to the $\boldsymbol{H}$ (curl)-topology by

$$
\boldsymbol{H}_{0}(\operatorname{curl}):={\overline{\mathcal{C}_{0}^{\infty}(\Omega)}}^{\|\cdot\|_{\boldsymbol{H}(\mathrm{curl})}} .
$$

It is well known that the Hilbert space $\boldsymbol{H}_{0}$ (curl) admits the following characterization:

$$
\boldsymbol{H}_{0}(\operatorname{curl})=\left\{\boldsymbol{q} \in \boldsymbol{H}(\operatorname{curl}) \mid(\boldsymbol{q}, \operatorname{curl} \boldsymbol{v})_{\boldsymbol{L}^{2}(\Omega)}=(\operatorname{curl} \boldsymbol{q}, \boldsymbol{v})_{\boldsymbol{L}^{2}(\Omega)} \forall \boldsymbol{v} \in \boldsymbol{H}(\operatorname{curl})\right\} .
$$

Let $\epsilon, \mu: \Omega \rightarrow \mathbb{R}^{3 \times 3}$ denote the electric permittivity and the magnetic permeability in the medium $\Omega$. They are assumed to be of class $L^{\infty}(\Omega)^{3 \times 3}$, symmetric and uniformly positive-definite in the sense that there exist constants $\underline{\epsilon}, \underline{\mu}>0$ such that

$$
\xi^{T} \epsilon(x) \xi \geq \underline{\epsilon}|\xi|^{2} \quad \text { and } \quad \xi^{T} \mu(x) \xi \geq \underline{\mu}|\xi|^{2} \quad \text { for a.e. } x \in \Omega \text { and all } \xi \in \mathbb{R}^{3} .
$$

For symmetric and uniformly positive definite matrix-valued function $\alpha \in L^{\infty}(\Omega)^{3 \times 3}$, let $\boldsymbol{L}_{\alpha}^{2}(\Omega)$ denote the weighted $\boldsymbol{L}^{2}(\Omega)$-space endowed with the weighted scalar product $(\alpha \cdot, \cdot)_{\boldsymbol{L}^{2}(\Omega)}$. Based on this notation, let us define the weighted product space

$$
\boldsymbol{X}:=\boldsymbol{L}_{\epsilon}^{2}(\Omega) \times \boldsymbol{L}_{\mu}^{2}(\Omega),
$$

equipped with the scalar product

$$
((\boldsymbol{e}, \boldsymbol{h}),(\boldsymbol{v}, \boldsymbol{w}))_{\boldsymbol{X}}=(\epsilon \boldsymbol{e}, \boldsymbol{v})_{\boldsymbol{L}^{2}(\Omega)}+(\mu \boldsymbol{h}, \boldsymbol{w})_{\boldsymbol{L}^{2}(\Omega)}, \quad \forall(\boldsymbol{e}, \boldsymbol{h}),(\boldsymbol{v}, \boldsymbol{w}) \in \boldsymbol{X} .
$$

Now, we introduce the (unbounded) Maxwell operator

$$
\mathcal{A}: D(\mathcal{A}) \subset \boldsymbol{X} \rightarrow \boldsymbol{X}, \quad \mathcal{A}:=-\left(\begin{array}{cc}
\epsilon & 0 \\
0 & \mu
\end{array}\right)^{-1}\left(\begin{array}{cc}
0 & - \text { curl } \\
\text { curl } & 0
\end{array}\right),
$$

with $D(\mathcal{A}):=\boldsymbol{H}_{0}(\mathbf{c u r l}) \times \boldsymbol{H}(\mathbf{c u r l})$. Due to the choice of the weighted Hilbert space $\boldsymbol{X}$ and (2.1), the Maxwell operator $\mathcal{A}: D(\mathcal{A}) \subset \boldsymbol{X} \rightarrow \boldsymbol{X}$ is skew-adjoint, i.e., $D\left(\mathcal{A}^{*}\right)=$ $D(\mathcal{A})$ and $\mathcal{A}^{*}=-\mathcal{A}$. Therefore, by virtue of Stone's theorem [18, Theorem 10.8, p. 41], $\mathcal{A}$ generates a strongly continuous group $\left\{\mathbb{T}_{t}\right\}_{t \in \mathbb{R}}$ of unitary operators on $\boldsymbol{X}$.

LEMma 2.1. Let $\left\{\mathbb{S}_{t}\right\}_{t \in \mathbb{R}}$ be a strongly continuous group of unitary operators on $\boldsymbol{X}$. Furthermore, suppose that $(\boldsymbol{e}, \boldsymbol{h}) \in \mathcal{C}([0, T], \boldsymbol{X}),\left(\boldsymbol{e}_{0}, \boldsymbol{h}_{0}\right) \in \boldsymbol{X}$ and $(\boldsymbol{w}, \hat{\boldsymbol{w}}) \in$ $L^{1}((0, T), \boldsymbol{X})$ satisfy the variation of constants formula

$$
(\boldsymbol{e}, \boldsymbol{h})(t)=\mathbb{S}_{t}\left(\boldsymbol{e}_{0}, \boldsymbol{h}_{0}\right)+\int_{0}^{t} \mathbb{S}_{t-s}(\boldsymbol{w}, \hat{\boldsymbol{w}})(s) d s, \quad \forall t \in[0, T] .
$$

Then, the following energy balance equality holds:

$$
\|(\boldsymbol{e}, \boldsymbol{h})(t)\|_{\boldsymbol{X}}^{2}=\left\|\left(\boldsymbol{e}_{0}, \boldsymbol{h}_{0}\right)\right\|_{\boldsymbol{X}}^{2}+2 \int_{0}^{t}((\boldsymbol{w}, \hat{\boldsymbol{w}})(s),(\boldsymbol{e}, \boldsymbol{h})(s))_{\boldsymbol{X}} d s, \quad \forall t \in[0, T] .
$$


Proof. As $\left\{\mathbb{S}_{t}\right\}_{t \in \mathbb{R}}$ is a strongly continuous group of unitary operators on $\boldsymbol{X}$, its infinitesimal generator $\mathcal{B}: D(\mathcal{B}) \subset \boldsymbol{X} \rightarrow \boldsymbol{X}$ is skew-adjoint (Stone's theorem). Since $D(\mathcal{B}) \subset \boldsymbol{X}$ and $\mathcal{C}_{0}^{\infty}((0, T), \boldsymbol{X}) \subset L^{1}((0, T), \boldsymbol{X})$ are dense, there exist $\left\{\left(\boldsymbol{e}_{n, 0}, \boldsymbol{h}_{n, 0}\right)\right\}_{n=1}^{\infty} \subset D(\mathcal{B})$ and $\left\{\left(\boldsymbol{w}_{n}, \hat{\boldsymbol{w}}_{n}\right)\right\}_{n=1}^{\infty} \subset \mathcal{C}_{0}^{\infty}((0, T), \boldsymbol{X})$ such that

$$
\lim _{n \rightarrow \infty}\left\|\left(\boldsymbol{e}_{n, 0}-\boldsymbol{e}_{0}, \boldsymbol{h}_{n, 0}-\boldsymbol{h}_{0}\right)\right\|_{\boldsymbol{X}}=0 \text { and } \lim _{n \rightarrow \infty}\left\|\left(\boldsymbol{w}_{n}-\boldsymbol{w}, \hat{\boldsymbol{w}}_{n}-\hat{\boldsymbol{w}}\right)\right\|_{L^{1}((0, T), \boldsymbol{X})}=0 .
$$

For every $n \in \mathbb{N}$, we define

$$
\left(\boldsymbol{e}_{n}, \boldsymbol{h}_{n}\right)(t):=\mathbb{S}_{t}\left(\boldsymbol{e}_{n, 0}, \boldsymbol{h}_{n, 0}\right)+\int_{0}^{t} \mathbb{S}_{t-s}\left(\boldsymbol{w}_{n}, \hat{\boldsymbol{w}}_{n}\right)(s) d s, \quad \forall t \in[0, T] .
$$

By definition and since $\left\{\mathbb{S}_{t}\right\}_{t \in \mathbb{R}}$ is unitary, we infer that

$$
\begin{aligned}
& \left\|\left(\boldsymbol{e}_{n}-\boldsymbol{e}, \boldsymbol{h}_{n}-\boldsymbol{h}\right)(t)\right\|_{\boldsymbol{X}} \\
& =\left\|\mathbb{S}_{t}\left(\boldsymbol{e}_{n, 0}-\boldsymbol{e}_{0}, \boldsymbol{h}_{n, 0}-\boldsymbol{h}_{0}\right)+\int_{0}^{t} \mathbb{S}_{t-s}\left(\boldsymbol{w}_{n}-\boldsymbol{w}, \hat{\boldsymbol{w}}_{n}-\hat{\boldsymbol{w}}\right)(s) d s\right\|_{\boldsymbol{X}} \\
& \leq\left\|\left(\boldsymbol{e}_{n, 0}-\boldsymbol{e}_{0}, \boldsymbol{h}_{n, 0}-\boldsymbol{h}_{0}\right)\right\|_{\boldsymbol{X}}+\left\|\left(\boldsymbol{w}_{n}-\boldsymbol{w}, \hat{\boldsymbol{w}}_{n}-\hat{\boldsymbol{w}}\right)\right\|_{L^{1}((0, t), \boldsymbol{X})}, \quad \forall t \in[0, T], \quad \forall n \in \mathbb{N} .
\end{aligned}
$$

It follows therefore for every $n \in \mathbb{N}$ that

$\left\|\left(\boldsymbol{e}_{n}-\boldsymbol{e}, \boldsymbol{h}_{n}-\boldsymbol{h}\right)\right\|_{\mathcal{C}([0, T], \boldsymbol{X})} \leq\left\|\left(\boldsymbol{e}_{n, 0}-\boldsymbol{e}_{0}, \boldsymbol{h}_{n, 0}-\boldsymbol{h}_{0}\right)\right\|_{\boldsymbol{X}}+\left\|\left(\boldsymbol{w}_{n}-\boldsymbol{w}, \hat{\boldsymbol{w}}_{n}-\hat{\boldsymbol{w}}\right)\right\|_{L^{1}((0, T), \boldsymbol{X})}$,

and so (2.5) implies

$$
\lim _{n \rightarrow \infty}\left\|\left(\boldsymbol{e}_{n}-\boldsymbol{e}, \boldsymbol{h}_{n}-\boldsymbol{h}\right)\right\|_{\mathcal{C}([0, T], \boldsymbol{X})}=0 .
$$

On the other hand, since $\left(\boldsymbol{e}_{n, 0}, \boldsymbol{h}_{n, 0}\right) \in D(\mathcal{B})$ and $\left(\boldsymbol{w}_{n}, \hat{\boldsymbol{w}}_{n}\right) \in \mathcal{C}_{0}^{\infty}((0, T), \boldsymbol{X})$, it holds for every $n \in \mathbb{N}$ that $\left(\boldsymbol{e}_{n}, \boldsymbol{h}_{n}\right) \in \mathcal{C}([0, T], D(\mathcal{B})) \cap \mathcal{C}^{1}([0, T], \boldsymbol{X})$, and it is exactly the solution of

$$
\left\{\begin{aligned}
\frac{d}{d t}\left(\boldsymbol{e}_{n}, \boldsymbol{h}_{n}\right)(t) & =\mathcal{B}\left(\boldsymbol{e}_{n}, \boldsymbol{h}_{n}\right)(t)+\left(\boldsymbol{w}_{n}, \hat{\boldsymbol{w}}_{n}\right)(t), \quad \forall t \in[0, T], \\
\left(\boldsymbol{e}_{n}, \boldsymbol{h}_{n}\right)(0) & =\left(\boldsymbol{e}_{n, 0}, \boldsymbol{h}_{n, 0}\right) .
\end{aligned}\right.
$$

See $[18$, Corollary 2.5, p. 107] for this classical result. Thus, for every $t \in[0, T]$ and $n \in \mathbb{N}$, it follows that

$$
\begin{aligned}
& \int_{0}^{t}\left(\frac{d}{d t}\left(\boldsymbol{e}_{n}, \boldsymbol{h}_{n}\right)(s),\left(\boldsymbol{e}_{n}, \boldsymbol{h}_{n}\right)(s)\right)_{\boldsymbol{X}} d s \\
& =\int_{0}^{t}\left(\mathcal{B}\left(\boldsymbol{e}_{n}, \boldsymbol{h}_{n}\right)(s),\left(\boldsymbol{e}_{n}, \boldsymbol{h}_{n}\right)(s)\right)_{\boldsymbol{X}} d s+\int_{0}^{t}\left(\left(\boldsymbol{w}_{n}, \hat{\boldsymbol{w}}_{n}\right)(s),\left(\boldsymbol{e}_{n}, \boldsymbol{h}_{n}\right)(s)\right)_{\boldsymbol{X}} d s \\
& =\int_{0}^{t}\left(\left(\boldsymbol{w}_{n}, \hat{\boldsymbol{w}}_{n}\right)(s),\left(\boldsymbol{e}_{n}, \boldsymbol{h}_{n}\right)(s)\right)_{\boldsymbol{X}} d s,
\end{aligned}
$$

since $\mathcal{B}$ is skew-adjoint. In conclusion, we obtain for every $t \in[0, T]$ and $n \in \mathbb{N}$ that

$$
\frac{1}{2}\left\|\left(\boldsymbol{e}_{n}, \boldsymbol{h}_{n}\right)(t)\right\|_{\boldsymbol{X}}^{2}-\frac{1}{2}\left\|\left(\boldsymbol{e}_{n, 0}, \boldsymbol{h}_{n, 0}\right)\right\|_{\boldsymbol{X}}^{2}=\int_{0}^{t}\left(\left(\boldsymbol{w}_{n}, \hat{\boldsymbol{w}}_{n}\right)(s),\left(\boldsymbol{e}_{n}, \boldsymbol{h}_{n}\right)(s)\right)_{\boldsymbol{X}} d s .
$$

Now, passing to the limit $n \rightarrow \infty$, the assertion follows from (2.5) and (2.6). 
3. Main results. In this section, we present our existence and uniqueness results for a general class of hyperbolic Maxwell variational inequalities of the second kind. The proof is given in Section 4 and Section 5. In the following, let $T \in \mathbb{R}^{+}$and

$$
\varphi: X \rightarrow \overline{\mathbb{R}}:=\mathbb{R} \cup\{+\infty\}
$$

be a proper, convex and lower semicontinuous (l.s.c.) function. We recall that the subdifferential $\partial \varphi: \boldsymbol{X} \rightarrow 2^{\boldsymbol{X}}$ is a set-valued operator, where, for every $(\boldsymbol{e}, \boldsymbol{h}) \in \boldsymbol{X}$, $\partial \varphi(\boldsymbol{e}, \boldsymbol{h})$ contains all subgradients of $\varphi$ at $(\boldsymbol{e}, \boldsymbol{h})$, i.e.,

$$
\begin{array}{r}
\partial \varphi(\boldsymbol{e}, \boldsymbol{h})=\left\{(\hat{\boldsymbol{e}}, \hat{\boldsymbol{h}}) \in \boldsymbol{X} \mid((\hat{\boldsymbol{e}}, \hat{\boldsymbol{h}}),(\boldsymbol{v}, \boldsymbol{w})-(\boldsymbol{e}, \boldsymbol{h}))_{\boldsymbol{X}} \leq \varphi(\boldsymbol{v}, \boldsymbol{w})-\varphi(\boldsymbol{e}, \boldsymbol{h})\right. \\
\forall(\boldsymbol{v}, \boldsymbol{w}) \in \boldsymbol{X}\} .
\end{array}
$$

Assumption 3.1. For every $M>0$, there exists a constant $C_{M}>0$ such that

$$
\|(\hat{\boldsymbol{e}}, \hat{\boldsymbol{h}})\|_{\boldsymbol{X}} \leq C_{M}, \quad \forall(\hat{\boldsymbol{e}}, \hat{\boldsymbol{h}}) \in \partial \varphi(\boldsymbol{e}, \boldsymbol{h}),
$$

for all $(\boldsymbol{e}, \boldsymbol{h}) \in \boldsymbol{X}$ satisfying $\|(\boldsymbol{e}, \boldsymbol{h})\|_{\boldsymbol{X}} \leq M$.

Theorem 3.2. Let $\varphi: \boldsymbol{X} \rightarrow \overline{\mathbb{R}}$ be a proper, convex and l.s.c. function satisfying $\partial \varphi(0,0) \neq \emptyset$ and Assumption 3.1. Then, for every $(\mathbf{f}, \mathbf{g}) \in L^{1}((0, T), \boldsymbol{X})$ and $\left(\boldsymbol{E}_{0}, \boldsymbol{H}_{0}\right) \in \boldsymbol{X}$, there exist unique pairs $(\boldsymbol{E}, \boldsymbol{H}) \in \mathcal{C}([0, T], \boldsymbol{X})$ and $(\hat{\boldsymbol{E}}, \hat{\boldsymbol{H}}) \in$ $L^{\infty}((0, T), \boldsymbol{X})$ such that

$$
\left\{\begin{array}{l}
\frac{d}{d t} \int_{\Omega} \epsilon \boldsymbol{E}(t) \cdot \boldsymbol{v}+\mu \boldsymbol{H}(t) \cdot \boldsymbol{w} d x+\int_{\Omega} \boldsymbol{E}(t) \cdot \operatorname{curl} \boldsymbol{w}-\boldsymbol{H}(t) \cdot \operatorname{curl} \boldsymbol{v} d x \\
+\int_{\Omega} \epsilon \hat{\boldsymbol{E}}(t) \cdot \boldsymbol{v}+\mu \hat{\boldsymbol{H}}(t) \cdot \boldsymbol{w} d x=\int_{\Omega} \mathbf{f}(t) \cdot \boldsymbol{v}+\mathbf{g}(t) \cdot \boldsymbol{w} d x, \\
\text { for a.e. } t \in(0, T) \text { and all }(\boldsymbol{v}, \boldsymbol{w}) \in \boldsymbol{H}_{0}(\operatorname{curl}) \times \boldsymbol{H}(\mathbf{c u r l}), \\
(\hat{\boldsymbol{E}}, \hat{\boldsymbol{H}})(t) \in \partial \varphi((\boldsymbol{E}, \boldsymbol{H})(t)) \text { for a.e. } t \in(0, T), \\
(\boldsymbol{E}, \boldsymbol{H})(0)=\left(\boldsymbol{E}_{0}, \boldsymbol{H}_{0}\right),
\end{array}\right.
$$

and, for every $(\boldsymbol{v}, \boldsymbol{w}) \in \boldsymbol{H}_{0}(\mathbf{c u r l}) \times \boldsymbol{H}(\mathbf{c u r l})$, the mapping $t \mapsto((\boldsymbol{E}, \boldsymbol{H})(t),(\boldsymbol{v}, \boldsymbol{w}))_{\boldsymbol{X}}$ is absolutely continuous from $[0, T]$ to $\mathbb{R}$. Moreover, $(\boldsymbol{E}, \boldsymbol{H})$ satisfies the Maxwell variational inequality

$$
\left\{\begin{array}{l}
\frac{d}{d t} \int_{\Omega} \epsilon \boldsymbol{E}(t) \cdot\left(\boldsymbol{v}-\frac{1}{2} \boldsymbol{E}(t)\right)+\mu \boldsymbol{H}(t) \cdot\left(\boldsymbol{w}-\frac{1}{2} \boldsymbol{H}(t)\right) d x \\
+\int_{\Omega} \boldsymbol{E}(t) \cdot \operatorname{curl} \boldsymbol{w}-\boldsymbol{H}(t) \cdot \mathbf{c u r l} \boldsymbol{v} d x+\varphi(\boldsymbol{v}, \boldsymbol{w})-\varphi((\boldsymbol{E}, \boldsymbol{H})(t)) \\
\geq \int_{\Omega} \mathbf{f}(t) \cdot(\boldsymbol{v}-\boldsymbol{E}(t)) d x+\int_{\Omega} \mathbf{g}(t) \cdot(\boldsymbol{w}-\boldsymbol{H}(t)) d x \\
\text { for a.e. } t \in(0, T) \text { and all }(\boldsymbol{v}, \boldsymbol{w}) \in \boldsymbol{H}_{0}(\operatorname{curl}) \times \boldsymbol{H}(\mathbf{c u r l}) \\
(\boldsymbol{E}, \boldsymbol{H})(0)=\left(\boldsymbol{E}_{0}, \boldsymbol{H}_{0}\right) .
\end{array}\right.
$$

Theorem 3.3. Let $\varphi: \boldsymbol{X} \rightarrow \overline{\mathbb{R}}$ be a proper, convex and l.s.c. function satisfying $\partial \varphi(0,0) \neq \emptyset$ and Assumption 3.1. Then, for every $(\mathbf{f}, \mathbf{g}) \in \mathcal{C}^{0,1}([0, T], \boldsymbol{X})$ and 
$\left(\boldsymbol{E}_{0}, \boldsymbol{H}_{0}\right) \in \boldsymbol{H}_{0}(\mathbf{c u r l}) \times \boldsymbol{H}(\mathbf{c u r l})$, the Maxwell variational inequality

$$
\left\{\begin{array}{l}
\int_{\Omega} \epsilon \frac{d}{d t} \boldsymbol{E}(t) \cdot(\boldsymbol{v}-\boldsymbol{E}(t))+\mu \frac{d}{d t} \boldsymbol{H}(t) \cdot(\boldsymbol{w}-\boldsymbol{H}(t)) d x \\
+\int_{\Omega} \operatorname{curl} \boldsymbol{E}(t) \cdot \boldsymbol{w}-\operatorname{curl} \boldsymbol{H}(t) \cdot \boldsymbol{v} d x+\varphi(\boldsymbol{v}, \boldsymbol{w})-\varphi((\boldsymbol{E}, \boldsymbol{H})(t)) \\
\geq \int_{\Omega} \mathbf{f}(t) \cdot(\boldsymbol{v}-\boldsymbol{E}(t)) d x+\int_{\Omega} \mathbf{g}(t) \cdot(\boldsymbol{w}-\boldsymbol{H}(t)) d x \\
\text { for a.e. } t \in(0, T) \text { and all }(\boldsymbol{v}, \boldsymbol{w}) \in \boldsymbol{X} \\
(\boldsymbol{E}, \boldsymbol{H})(0)=\left(\boldsymbol{E}_{0}, \boldsymbol{H}_{0}\right)
\end{array}\right.
$$

admits a unique solution $(\boldsymbol{E}, \boldsymbol{H}) \in L^{\infty}\left((0, T), \boldsymbol{H}_{0}(\mathbf{c u r l}) \times \boldsymbol{H}(\mathbf{c u r l})\right) \cap W^{1, \infty}((0, T), \boldsymbol{X})$, which at the same time solves both (3.2) and (3.3).

REMARK 3.4. If $\varphi \equiv 0$ and $\mathbf{g} \equiv 0$, then Theorem 3.2 and Theorem 3.3 are nothing but the classical existence and uniqueness results (cf. Leis [13]) for the weak and strong solutions to the Maxwell equations

$$
\begin{cases}\epsilon \frac{d}{d t} \boldsymbol{E}-\operatorname{curl} \boldsymbol{H}=\mathbf{f} & \text { in } \Omega \times(0, T), \quad \boldsymbol{E}(0)=\boldsymbol{E}_{0} \text { in } \Omega, \\ \mu \frac{d}{d t} \boldsymbol{H}+\operatorname{curl} \boldsymbol{E}=0 & \text { in } \Omega \times(0, T), \quad \boldsymbol{H}(0)=\boldsymbol{H}_{0} \text { in } \Omega .\end{cases}
$$

4. Proof of Theorem 3.2. We split our proof for Theorem 3.2 into three parts:

(i) In Section 4.1, we prove the existence result for (3.2).

(ii) In Section 4.2, we prove the uniqueness result for (3.2).

(iii) In Section 4.3, we show that the solution of (3.2) solves (3.3).

Let us begin by recalling the classical Moreau-Yosida approximation theory. For every $\lambda>0$, we define the Moreau-Yosida approximation

$$
\varphi_{\lambda}(\boldsymbol{e}, \boldsymbol{h}):=\inf _{(\boldsymbol{v}, \boldsymbol{w}) \in \boldsymbol{X}} \frac{1}{2 \lambda}\|(\boldsymbol{v}, \boldsymbol{w})-(\boldsymbol{e}, \boldsymbol{h})\|_{\boldsymbol{X}}^{2}+\varphi(\boldsymbol{v}, \boldsymbol{w})
$$

Since $\varphi: \boldsymbol{X} \rightarrow \overline{\mathbb{R}}$ is a proper, convex and l.s.c. function, Moreau's theorem [22, Proposition 1.8, p. 162] implies that, for every $\lambda>0, \varphi_{\lambda}: \boldsymbol{X} \rightarrow \mathbb{R}$ is a convex and Fréchet differentiable function satisfying

$$
\varphi_{\lambda}(\boldsymbol{v}, \boldsymbol{w})=\varphi\left(J_{\lambda}(\boldsymbol{v}, \boldsymbol{w})\right)+\frac{\lambda}{2}\left\|\Phi_{\lambda}(\boldsymbol{v}, \boldsymbol{w})\right\|_{\boldsymbol{X}}^{2} \quad \text { and } \quad \varphi_{\lambda}^{\prime}(\boldsymbol{v}, \boldsymbol{w})=\Phi_{\lambda}(\boldsymbol{v}, \boldsymbol{w}),
$$

for all $(\boldsymbol{v}, \boldsymbol{w}) \in \boldsymbol{X}$. Here, $J_{\lambda}: \boldsymbol{X} \rightarrow \boldsymbol{X}$ and $\Phi_{\lambda}: \boldsymbol{X} \rightarrow \boldsymbol{X}$ denote, respectively, the resolvent and the Yosida approximation of the subdifferential $\partial \varphi$, i.e.,

$$
J_{\lambda}=\left(I_{d}+\lambda \partial \varphi\right)^{-1} \quad \text { and } \quad \Phi_{\lambda}=\lambda^{-1}\left(I_{d}-J_{\lambda}\right)
$$

where $I_{d}: \boldsymbol{X} \rightarrow \boldsymbol{X}$ denotes the identity operator. Since $\varphi$ is proper, convex, and lower semicontinuous, the subdifferential $\partial \varphi: \boldsymbol{X} \rightarrow 2^{\boldsymbol{X}}$ is maximal monotone (maccretive). See, e.g, [22, Proposition 1.5, p. 157] for this well-known result. For this reason, the Yosida approximation $\Phi_{\lambda}: \boldsymbol{X} \rightarrow \boldsymbol{X}$ is maximal monotone and Lipschitzcontinuous with Lipschitz constant $L_{\lambda}=\lambda^{-1}$, and the resolvent $J_{\lambda}: \boldsymbol{X} \rightarrow \boldsymbol{X}$ is Lipschitz-continuous with Lipschitz constant $L=1$ (see [1, Theorem 3.5.9, p. 111]). 
4.1. Existence. Let $(\mathbf{f}, \mathbf{g}) \in L^{1}((0, T), \boldsymbol{X})$ and $\left(\boldsymbol{E}_{0}, \boldsymbol{H}_{0}\right) \in \boldsymbol{X}$. Furthermore, let $\left\{\lambda_{n}\right\}_{n=1}^{\infty}$ be a null sequence of positive real numbers, and we consider the following integral equation: For every $n \in \mathbb{N}$, find $\left(\boldsymbol{E}_{n}, \boldsymbol{H}_{n}\right) \in \mathcal{C}([0, T], \boldsymbol{X})$ such that

$$
\left(\boldsymbol{E}_{n}, \boldsymbol{H}_{n}\right)(t)=\mathbb{T}_{t}\left(\boldsymbol{E}_{0}, \boldsymbol{H}_{0}\right)+\int_{0}^{t} \mathbb{T}_{t-s}\left(\left(\epsilon^{-1} \mathbf{f}, \mu^{-1} \mathbf{g}\right)(s)-\Phi_{\lambda_{n}}\left(\left(\boldsymbol{E}_{n}, \boldsymbol{H}_{n}\right)(s)\right)\right) d s,
$$

$$
\forall t \in[0, T] .
$$

Since for every $\lambda>0$ the Yosida approximation $\Phi_{\lambda}: \boldsymbol{X} \rightarrow \boldsymbol{X}$ is Lipschitz-continous, a classical contraction argument [18, Theorem 1.2, p. 184] implies that, for every $n \in \mathbb{N}$, the integral equation (4.4) admits a unique solution $\left(\boldsymbol{E}_{n}, \boldsymbol{H}_{n}\right) \in \mathcal{C}([0, T], \boldsymbol{X})$. Thanks to the Lipschitz continuity of $J_{\lambda_{n}}: \boldsymbol{X} \rightarrow \boldsymbol{X}$ and $\Phi_{\lambda_{n}}: \boldsymbol{X} \rightarrow \boldsymbol{X}$, we have that

$$
J_{\lambda_{n}}\left(\boldsymbol{E}_{n}, \boldsymbol{H}_{n}\right), \Phi_{\lambda_{n}}\left(\boldsymbol{E}_{n}, \boldsymbol{H}_{n}\right) \in \mathcal{C}([0, T], \boldsymbol{X}), \quad \forall n \in \mathbb{N} .
$$

Let us show that the sequences $\left\{\left(\boldsymbol{E}_{n}, \boldsymbol{H}_{n}\right)\right\}_{n=1}^{\infty},\left\{J_{\lambda_{n}}\left(\boldsymbol{E}_{n}, \boldsymbol{H}_{n}\right)\right\}_{n=1}^{\infty},\left\{\Phi_{\lambda_{n}}\left(\boldsymbol{E}_{n}, \boldsymbol{H}_{n}\right)\right\}_{n=1}^{\infty}$ are bounded in $\mathcal{C}([0, T], \boldsymbol{X})$.

Since $\left\{\mathbb{T}_{t}\right\}_{t \in \mathbb{R}}$ is a strongly continuous group of unitary operators on $\boldsymbol{X}$, we may apply the energy balance equality (Lemma 2.1) to (4.4) and obtain that

$$
\begin{array}{r}
\left\|\left(\boldsymbol{E}_{n}, \boldsymbol{H}_{n}\right)(t)\right\|_{\boldsymbol{X}}^{2}=\left\|\left(\boldsymbol{E}_{0}, \boldsymbol{H}_{0}\right)\right\|_{\boldsymbol{X}}^{2}+2 \int_{0}^{t}\left(\left(\epsilon^{-1} \mathbf{f}, \mu^{-1} \mathbf{g}\right)(s),\left(\boldsymbol{E}_{n}, \boldsymbol{H}_{n}\right)(s)\right)_{\boldsymbol{X}} \\
-\left(\Phi_{\lambda_{n}}\left(\left(\boldsymbol{E}_{n}, \boldsymbol{H}_{n}\right)(s)\right),\left(\boldsymbol{E}_{n}, \boldsymbol{H}_{n}\right)(s)\right)_{\boldsymbol{X}} d s \\
=\left\|\left(\boldsymbol{E}_{0}, \boldsymbol{H}_{0}\right)\right\|_{\boldsymbol{X}}^{2}+2 \int_{0}^{t}\left(\left(\epsilon^{-1} \mathbf{f}, \mu^{-1} \mathbf{g}\right)(s),\left(\boldsymbol{E}_{n}, \boldsymbol{H}_{n}\right)(s)\right)_{\boldsymbol{X}} \\
-\left(\Phi_{\lambda_{n}}\left(\left(\boldsymbol{E}_{n}, \boldsymbol{H}_{n}\right)(s)\right)-\Phi_{\lambda_{n}}(0,0),\left(\boldsymbol{E}_{n}, \boldsymbol{H}_{n}\right)(s)\right)_{\boldsymbol{X}}-\left(\Phi_{\lambda_{n}}(0,0),\left(\boldsymbol{E}_{n}, \boldsymbol{H}_{n}\right)(s)\right)_{\boldsymbol{X}} d s,
\end{array}
$$

for all $t \in[0, T]$ and $n \in \mathbb{N}$. The monotonicity of the Yosida approximation implies

$$
\left(\Phi_{\lambda_{n}}\left(\left(\boldsymbol{E}_{n}, \boldsymbol{H}_{n}\right)(s)\right)-\Phi_{\lambda_{n}}(0,0),\left(\boldsymbol{E}_{n}, \boldsymbol{H}_{n}\right)(s)\right)_{\boldsymbol{X}} \geq 0, \quad \forall s \in[0, T], \quad \forall n \in \mathbb{N},
$$

from which it follows that

$$
\begin{aligned}
& \left\|\left(\boldsymbol{E}_{n}, \boldsymbol{H}_{n}\right)(t)\right\|_{\boldsymbol{X}}^{2} \leq\left\|\left(\boldsymbol{E}_{0}, \boldsymbol{H}_{0}\right)\right\|_{\boldsymbol{X}}^{2} \\
& \quad+2 \int_{0}^{t}\left(\left\|\left(\epsilon^{-1} \mathbf{f}, \mu^{-1} \mathbf{g}\right)(s)\right\|_{\boldsymbol{X}}+\left\|\Phi_{\lambda_{n}}(0,0)\right\|_{\boldsymbol{X}}\right)\left\|\left(\boldsymbol{E}_{n}, \boldsymbol{H}_{n}\right)(s)\right\|_{\boldsymbol{X}} d s, \quad \forall t \in[0, T], \quad \forall n \in \mathbb{N} .
\end{aligned}
$$

The assumption $\partial \varphi(0,0) \neq \emptyset$ implies that $\left\|\Phi_{\lambda_{n}}(0,0)\right\|_{\boldsymbol{X}}$ converges as $n \rightarrow \infty$ (see [22, Theorem 1.1(c), p. 161]). For this reason, we obtain from the above inequality that the sequence $\left\{\left(\boldsymbol{E}_{n}, \boldsymbol{H}_{n}\right)\right\}_{n=1}^{\infty} \subset \mathcal{C}([0, T], \boldsymbol{X})$ is bounded. Next, due to the Lipschitzcontinuity of the resolvent $J_{\lambda}: \boldsymbol{X} \rightarrow \boldsymbol{X}$ with Lipschitz constant $L=1$, we obtain

$$
\left\|J_{\lambda_{n}}\left(\left(\boldsymbol{E}_{n}, \boldsymbol{H}_{n}\right)(t)\right)\right\|_{\boldsymbol{X}} \leq\left\|\left(\boldsymbol{E}_{n}, \boldsymbol{H}_{n}\right)(t)\right\|_{\boldsymbol{X}}+\left\|J_{\lambda_{n}}(0,0)\right\|_{\boldsymbol{X}}, \quad \forall t \in[0, T], \quad \forall n \in \mathbb{N} .
$$

Thus, since $\lim _{n \rightarrow \infty}\left\|J_{\lambda_{n}}(0,0)\right\|_{\boldsymbol{X}}=0$ (due to $\partial \varphi(0,0) \neq \emptyset$ ), the above inequality together with the boundedness of $\left\{\left(\boldsymbol{E}_{n}, \boldsymbol{H}_{n}\right)\right\}_{n=1}^{\infty} \subset \mathcal{C}([0, T], \boldsymbol{X})$ implies that $\left\{J_{\lambda_{n}}\left(\boldsymbol{E}_{n}, \boldsymbol{H}_{n}\right)\right\}_{n=1}^{\infty} \subset \mathcal{C}([0, T], \boldsymbol{X})$ is bounded. Furthermore, by the definition of the resolvent and the Yosida approximation (4.3), we have that (see [1, Theorem 3.5.9]):

$$
\Phi_{\lambda_{n}}\left(\left(\boldsymbol{E}_{n}, \boldsymbol{H}_{n}\right)(s)\right) \in \partial \varphi\left(J_{\lambda_{n}}\left(\left(\boldsymbol{E}_{n}, \boldsymbol{H}_{n}\right)(s)\right)\right), \quad \forall s \in[0, T], \quad \forall n \in \mathbb{N} .
$$

Thus, by Assumption 3.1 and the boundedness of $\left\{J_{\lambda_{n}}\left(\boldsymbol{E}_{n}, \boldsymbol{H}_{n}\right)\right\}_{n=1}^{\infty} \subset \mathcal{C}([0, T], \boldsymbol{X})$, (4.5) implies that $\left\{\Phi_{\lambda_{n}}\left(\boldsymbol{E}_{n}, \boldsymbol{H}_{n}\right)\right\}_{n=1}^{\infty} \subset \mathcal{C}([0, T], \boldsymbol{X})$ is bounded. In conclusion, the 
three sequences $\left\{\left(\boldsymbol{E}_{n}, \boldsymbol{H}_{n}\right)\right\}_{n=1}^{\infty},\left\{J_{\lambda_{n}}\left(\boldsymbol{E}_{n}, \boldsymbol{H}_{n}\right)\right\}_{n=1}^{\infty},\left\{\Phi_{\lambda_{n}}\left(\boldsymbol{E}_{n}, \boldsymbol{H}_{n}\right)\right\}_{n=1}^{\infty}$ are bounded in $\mathcal{C}([0, T], \boldsymbol{X})$. Therefore, we can select a subsequence of $\left\{\lambda_{n}\right\}_{n=1}^{\infty}$, which we denote again by $\left\{\lambda_{n}\right\}_{n=1}^{\infty}$, such that

$$
\begin{array}{ll}
\left(\boldsymbol{E}_{n}, \boldsymbol{H}_{n}\right) \rightarrow(\boldsymbol{E}, \boldsymbol{H}) & \text { weakly star in } L^{\infty}((0, T), \boldsymbol{X}) \text { as } n \rightarrow \infty, \\
\Phi_{\lambda_{n}}\left(\boldsymbol{E}_{n}, \boldsymbol{H}_{n}\right) \rightarrow(\hat{\boldsymbol{E}}, \hat{\boldsymbol{H}}) & \text { weakly star in } L^{\infty}((0, T), \boldsymbol{X}) \text { as } n \rightarrow \infty, \\
\left(I_{d}-J_{\lambda_{n}}\right)\left(\boldsymbol{E}_{n}, \boldsymbol{H}_{n}\right) \rightarrow 0 & \text { strongly in } \mathcal{C}([0, T], \boldsymbol{X}) \text { as } n \rightarrow \infty, \\
J_{\lambda_{n}}\left(\boldsymbol{E}_{n}, \boldsymbol{H}_{n}\right) \rightarrow(\boldsymbol{E}, \boldsymbol{H}) & \text { weakly star in } L^{\infty}((0, T), \boldsymbol{X}) \text { as } n \rightarrow \infty,
\end{array}
$$

for some $(\boldsymbol{E}, \boldsymbol{H})(\hat{\boldsymbol{E}}, \hat{\boldsymbol{H}}) \in L^{\infty}((0, T), \boldsymbol{X})$. We note that (4.8) follows from the boundedness of $\left\{\Phi_{\lambda_{n}}\left(\boldsymbol{E}_{n}, \boldsymbol{H}_{n}\right)\right\}_{n=1}^{\infty} \subset \mathcal{C}([0, T], \boldsymbol{X})$ and the definition of the Yosida approximation $\Phi_{\lambda}=\lambda^{-1}\left(I_{d}-J_{\lambda}\right)$. Moreover, (4.9) follows from (4.8) and (4.6).

Passing to the limit $n \rightarrow \infty$ in (4.4), we obtain from (4.6) and (4.7) that

$$
(\boldsymbol{E}, \boldsymbol{H})(t)=\mathbb{T}_{t}\left(\boldsymbol{E}_{0}, \boldsymbol{H}_{0}\right)+\int_{0}^{t} \mathbb{T}_{t-s}\left(\left(\epsilon^{-1} \mathbf{f}, \mu^{-1} \mathbf{g}\right)(s)-(\hat{\boldsymbol{E}}, \hat{\boldsymbol{H}})(s)\right) d s, \forall t \in[0, T] .
$$

and for every $t \in[0, T]$

$$
\left(\boldsymbol{E}_{n}, \boldsymbol{H}_{n}\right)(t) \rightarrow(\boldsymbol{E}, \boldsymbol{H})(t) \text { weakly in } \boldsymbol{X} \text { as } n \rightarrow \infty .
$$

Now, employing the classical result by Ball [3], the solution of (4.10) satisfies

$$
\left\{\begin{array}{l}
\frac{d}{d t}((\boldsymbol{E}, \boldsymbol{H})(t),(\boldsymbol{v}, \boldsymbol{w}))_{\boldsymbol{X}}-\left((\boldsymbol{E}, \boldsymbol{H})(t), \mathcal{A}^{*}(\boldsymbol{v}, \boldsymbol{w})\right)_{\boldsymbol{X}}=\left(\left(\epsilon^{-1} \mathbf{f}, \mu^{-1} \mathbf{g}\right)(t)\right. \\
\quad-(\hat{\boldsymbol{E}}, \hat{\boldsymbol{H}})(t),(\boldsymbol{v}, \boldsymbol{w}))_{\boldsymbol{X}}, \\
\text { for a.e. } t \in(0, T) \text { and all }(\boldsymbol{v}, \boldsymbol{w}) \in D\left(\mathcal{A}^{*}\right), \\
(\boldsymbol{E}, \boldsymbol{H})(0)=\left(\boldsymbol{E}_{0}, \boldsymbol{H}_{0}\right),
\end{array}\right.
$$

and, for every $(\boldsymbol{v}, \boldsymbol{w}) \in D\left(\mathcal{A}^{*}\right)$, the mapping $t \mapsto((\boldsymbol{E}, \boldsymbol{H})(t),(\boldsymbol{v}, \boldsymbol{w}))_{\boldsymbol{X}}$ is absolutely continuous from $[0, T]$ to $\mathbb{R}$.

Since the Maxwell operator $\mathcal{A}$ is skew adjoint, i.e., $D\left(\mathcal{A}^{*}\right)=D(\mathcal{A})=\boldsymbol{H}_{0}(\mathbf{c u r l}) \times$ $\boldsymbol{H}(\mathbf{c u r l})$ and $\mathcal{A}^{*}=-\mathcal{A}$ (see (2.4) on p. 2 for its definition), we see that (4.12) is equivalent to

$$
\left\{\begin{array}{l}
\frac{d}{d t} \int_{\Omega} \epsilon \boldsymbol{E}(t) \cdot \boldsymbol{v}+\mu \boldsymbol{H}(t) \cdot \boldsymbol{w} d x+\int_{\Omega} \boldsymbol{E}(t) \cdot \operatorname{curl} \boldsymbol{w}-\boldsymbol{H}(t) \cdot \operatorname{curl} \boldsymbol{v} d x \\
+\int_{\Omega} \epsilon \hat{\boldsymbol{E}}(t) \cdot \boldsymbol{v}+\mu \hat{\boldsymbol{H}}(t) \cdot \boldsymbol{w} d x=\int_{\Omega} \mathbf{f}(t) \cdot \boldsymbol{v}+\mathbf{g}(t) \cdot \boldsymbol{w} d x \\
\text { for a.e. } t \in(0, T) \text { and all }(\boldsymbol{v}, \boldsymbol{w}) \in \boldsymbol{H}_{0}(\operatorname{curl}) \times \boldsymbol{H}(\operatorname{curl}) \\
(\boldsymbol{E}, \boldsymbol{H})(0)=\left(\boldsymbol{E}_{0}, \boldsymbol{H}_{0}\right)
\end{array}\right.
$$

For this reason, if we can prove that

$$
(\hat{\boldsymbol{E}}, \hat{\boldsymbol{H}})(t) \in \partial \varphi((\boldsymbol{E}, \boldsymbol{H})(t)) \text { for a.e. } t \in(0, T),
$$

then we see that $(\boldsymbol{E}, \boldsymbol{H}) \in \mathcal{C}([0, T], \boldsymbol{X})$ is a solution to (3.2). To this aim, we introduce the set $B \subset L^{2}((0, T), \boldsymbol{X}) \times L^{2}((0, T), \boldsymbol{X})$ defined as follows:

$$
((\boldsymbol{e}, \boldsymbol{h}),(\hat{\boldsymbol{e}}, \hat{\boldsymbol{h}})) \in B \quad \Leftrightarrow \quad(\hat{\boldsymbol{e}}, \hat{\boldsymbol{h}})(t) \in \partial \varphi((\boldsymbol{e}, \boldsymbol{h})(t)) \text { for a.e. } t \in(0, T) .
$$


By definition, we see that (4.14) is nothing but

$$
((\boldsymbol{E}, \boldsymbol{H}),(\hat{\boldsymbol{E}}, \hat{\boldsymbol{H}})) \in B .
$$

Therefore, we have to show that (4.16) is satisfied.

Since $\partial \varphi: \boldsymbol{X} \rightarrow 2^{\boldsymbol{X}}$ is monotone, the set $B$ is monotone, i.e.,

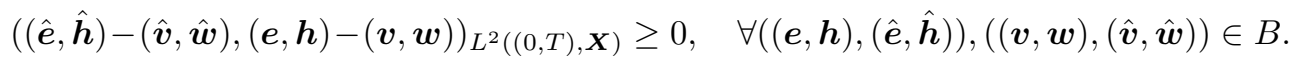

Let us now show that $B$ is maximal monotone (m-accretive), i.e., we have to show that, for every $\lambda>0$, it holds that

$$
\operatorname{Range}\left(I_{d}+\lambda B\right):=\{(\boldsymbol{e}, \boldsymbol{h})+\lambda(\hat{\boldsymbol{e}}, \hat{\boldsymbol{h}}) \mid((\boldsymbol{e}, \boldsymbol{h}),(\hat{\boldsymbol{e}}, \hat{\boldsymbol{h}})) \in B\}=L^{2}((0, T), \boldsymbol{X}) .
$$

Let $\lambda>0$. To show (4.17), we take an arbitrarily fixed function $(\boldsymbol{v}, \boldsymbol{w}) \in L^{2}((0, T), \boldsymbol{X})$ and define

$$
(\boldsymbol{e}, \boldsymbol{h})(t):=J_{\lambda}((\boldsymbol{v}, \boldsymbol{w})(t))=\left(I_{d}+\lambda \partial \varphi\right)^{-1}(\boldsymbol{v}, \boldsymbol{w})(t) \quad \text { for a.e. } t \in(0, T) .
$$

According to the definition (4.15), we see that (4.17) is valid, if $(\boldsymbol{e}, \boldsymbol{h}) \in L^{2}((0, T), \boldsymbol{X})$. Indeed, due to the Lipschitz continuity of $J_{\lambda}: \boldsymbol{X} \rightarrow \boldsymbol{X}$ (with Lipschitz constant $L=1)$, we obtain that $(\boldsymbol{e}, \boldsymbol{h})=J_{\lambda}(\boldsymbol{v}, \boldsymbol{w})$ is measurable and

$$
\begin{aligned}
\|(\boldsymbol{e}, \boldsymbol{h})(t)\|_{\boldsymbol{X}} & \leq\left\|J_{\lambda}((\boldsymbol{v}, \boldsymbol{w})(t))-J_{\lambda}(0,0)\right\|_{\boldsymbol{X}}+\left\|J_{\lambda}(0,0)\right\|_{\boldsymbol{X}} \\
& \leq\|(\boldsymbol{v}, \boldsymbol{w})(t)\|_{\boldsymbol{X}}+\left\|J_{\lambda}(0,0)\right\|_{\boldsymbol{X}} \quad \text { for a.e. } t \in(0, T) .
\end{aligned}
$$

Since $(\boldsymbol{v}, \boldsymbol{w}) \in L^{2}((0, T), \boldsymbol{X})$, it follows therefore that $(\boldsymbol{e}, \boldsymbol{h}) \in L^{2}((0, T), \boldsymbol{X})$. In conclusion, $B$ is maximal monotone.

Now, making use of the energy balance equality (Lemma 2.1) in (4.10) and (4.4), we obtain that

$$
\begin{aligned}
& \|(\boldsymbol{E}, \boldsymbol{H})(T)\|_{\boldsymbol{X}}^{2}=\left\|\left(\boldsymbol{E}_{0}, \boldsymbol{H}_{0}\right)\right\|_{\boldsymbol{X}}^{2}+2 \int_{0}^{T}\left(\left(\epsilon^{-1} \mathbf{f}, \mu^{-1} \mathbf{g}\right)(t)-(\hat{\boldsymbol{E}}, \hat{\boldsymbol{H}})(t),(\boldsymbol{E}, \boldsymbol{H})(t)\right)_{\boldsymbol{X}} d t \\
& \left\|\left(\boldsymbol{E}_{n}, \boldsymbol{H}_{n}\right)(T)\right\|_{\boldsymbol{X}}^{2}=\left\|\left(\boldsymbol{E}_{0}, \boldsymbol{H}_{0}\right)\right\|_{\boldsymbol{X}}^{2} \\
& \quad+2 \int_{0}^{T}\left(\left(\epsilon^{-1} \mathbf{f}, \mu^{-1} \mathbf{g}\right)(t)-\Phi_{\lambda_{n}}\left(\left(\boldsymbol{E}_{n}, \boldsymbol{H}_{n}\right)(t)\right),\left(\boldsymbol{E}_{n}, \boldsymbol{H}_{n}\right)(t)\right)_{\boldsymbol{X}} d t, \quad \forall n \in \mathbb{N} .
\end{aligned}
$$

Combining these two identities results in

$$
\begin{array}{r}
2 \int_{0}^{T}\left(\Phi_{\lambda_{n}}\left(\left(\boldsymbol{E}_{n}, \boldsymbol{H}_{n}\right)(t)\right),\left(\boldsymbol{E}_{n}, \boldsymbol{H}_{n}\right)(t)\right)_{\boldsymbol{X}} d t=-\left\|\left(\boldsymbol{E}_{n}, \boldsymbol{H}_{n}\right)(T)\right\|_{\boldsymbol{X}}^{2}+\left\|\left(\boldsymbol{E}_{0}, \boldsymbol{H}_{0}\right)\right\|_{\boldsymbol{X}}^{2} \\
+2 \int_{0}^{T}\left(\left(\epsilon^{-1} \mathbf{f}, \mu^{-1} \mathbf{g}\right)(t),\left(\boldsymbol{E}_{n}, \boldsymbol{H}_{n}\right)(t)\right)_{\boldsymbol{L}^{2}(\Omega)} d t \\
=-\left\|\left(\boldsymbol{E}_{n}, \boldsymbol{H}_{n}\right)(T)\right\|_{\boldsymbol{X}}^{2}+\|(\boldsymbol{E}, \boldsymbol{H})(T)\|_{\boldsymbol{X}}^{2}+2 \int_{0}^{T}((\hat{\boldsymbol{E}}, \hat{\boldsymbol{H}})(t),(\boldsymbol{E}, \boldsymbol{H})(t))_{\boldsymbol{X}} d t \\
+2 \int_{0}^{T}\left(\left(\epsilon^{-1} \mathbf{f}, \mu^{-1} \mathbf{g}\right)(t),\left(\boldsymbol{E}_{n}, \boldsymbol{H}_{n}\right)(t)-(\boldsymbol{E}, \boldsymbol{H})(t)\right)_{\boldsymbol{L}^{2}(\Omega)} d t
\end{array}
$$


Then, employing (4.8) in the above identity, we infer that

$$
\begin{aligned}
& 2 \liminf _{n \rightarrow \infty} \int_{0}^{T}\left(\Phi_{\lambda_{n}}\left(\left(\boldsymbol{E}_{n}, \boldsymbol{H}_{n}\right)(t)\right), J_{\lambda_{n}}\left(\left(\boldsymbol{E}_{n}, \boldsymbol{H}_{n}\right)(t)\right)\right)_{\boldsymbol{X}} d t \\
& =2 \liminf _{n \rightarrow \infty} \int_{0}^{T}\left(\Phi_{\lambda_{n}}\left(\left(\boldsymbol{E}_{n}, \boldsymbol{H}_{n}\right)(t)\right),\left(\boldsymbol{E}_{n}, \boldsymbol{H}_{n}\right)(t)\right)_{\boldsymbol{X}} d t \\
& \leq 2 \limsup _{n \rightarrow \infty} \int_{0}^{T}\left(\Phi_{\lambda_{n}}\left(\left(\boldsymbol{E}_{n}, \boldsymbol{H}_{n}\right)(t)\right),\left(\boldsymbol{E}_{n}, \boldsymbol{H}_{n}\right)(t)\right)_{\boldsymbol{X}} d t \\
& \leq \limsup _{n \rightarrow \infty}\left(-\left\|\left(\boldsymbol{E}_{n}, \boldsymbol{H}_{n}\right)(T)\right\|_{\boldsymbol{X}}^{2}\right)+\|(\boldsymbol{E}, \boldsymbol{H})(T)\|_{\boldsymbol{X}}^{2}+2 \int_{0}^{T}((\hat{\boldsymbol{E}}, \hat{\boldsymbol{H}})(t),(\boldsymbol{E}, \boldsymbol{H})(t))_{\boldsymbol{X}} d t \\
& \quad+2 \limsup _{n \rightarrow \infty} \int_{0}^{T}\left(\left(\epsilon^{-1} \mathbf{f}, \mu^{-1} \mathbf{g}\right)(t),\left(\boldsymbol{E}_{n}, \boldsymbol{H}_{n}\right)(t)-(\boldsymbol{E}, \boldsymbol{H})(t)\right)_{\boldsymbol{L}^{2}(\Omega)} d t \\
& =-\liminf _{n \rightarrow \infty}\left\|\left(\boldsymbol{E}_{n}, \boldsymbol{H}_{n}\right)(T)\right\|_{\boldsymbol{X}}^{2}+\|(\boldsymbol{E}, \boldsymbol{H})(T)\|_{\boldsymbol{X}}^{2}+2 \int_{0}^{T}((\hat{\boldsymbol{E}}, \hat{\boldsymbol{H}})(t),(\boldsymbol{E}, \boldsymbol{H})(t))_{\boldsymbol{X}} d t \\
& \quad+2 \limsup _{n \rightarrow \infty} \int_{0}^{T}\left(\left(\epsilon^{-1} \mathbf{f}, \mu^{-1} \mathbf{g}\right)(t),\left(\boldsymbol{E}_{n}, \boldsymbol{H}_{n}\right)(t)-(\boldsymbol{E}, \boldsymbol{H})(t)\right)_{\boldsymbol{L}^{2}(\Omega)} d t .
\end{aligned}
$$

It follows therefore from the weak convergence properties (4.6) and (4.11) with $t=T$ that

$$
\begin{array}{r}
\liminf _{n \rightarrow \infty} \int_{0}^{T}\left(\Phi_{\lambda_{n}}\left(\left(\boldsymbol{E}_{n}, \boldsymbol{H}_{n}\right)(t)\right), J_{\lambda_{n}}\left(\boldsymbol{E}_{n}, \boldsymbol{H}_{n}\right)(t)\right)_{\boldsymbol{X}} d t \\
\leq \int_{0}^{T}((\hat{\boldsymbol{E}}, \hat{\boldsymbol{H}})(t),(\boldsymbol{E}, \boldsymbol{H})(t))_{\boldsymbol{X}} d t .
\end{array}
$$

On the other hand, according to (4.5) and by the definition (4.15), it holds that

$$
\left(J_{\lambda_{n}}\left(\boldsymbol{E}_{n}, \boldsymbol{H}_{n}\right), \Phi_{\lambda_{n}}\left(\boldsymbol{E}_{n}, \boldsymbol{H}_{n}\right)\right) \in B, \quad \forall n \in \mathbb{N} .
$$

Due to the maximal monotonicity property of $B$, the inclusion (4.19), the weak convergence properties (4.7) and (4.9), we obtain from the inequality (4.18) that (4.16) is satisfied. This is due to the well-known monotonicity trick (see [22, Proposition 1.6 p. 159]). In conclusion, $(\boldsymbol{E}, \boldsymbol{H}) \in \mathcal{C}([0, T], \boldsymbol{X})$ satisfies (4.13)-(4.14), and hence it is a solution of (3.2).

4.2. Uniqueness. Let $(\mathbf{f}, \mathbf{g}) \in L^{1}((0, T), \boldsymbol{X})$ and $\left(\boldsymbol{E}_{0}, \boldsymbol{H}_{0}\right) \in \boldsymbol{X}$. Suppose that $\left(\boldsymbol{E}^{(1)}, \boldsymbol{H}^{(1)}\right),\left(\boldsymbol{E}^{(2)}, \boldsymbol{H}^{(2)}\right) \in \mathcal{C}([0, T], \boldsymbol{X})$ satisfy (3.2) and, for every $(\boldsymbol{v}, \boldsymbol{w}) \in$ $\boldsymbol{H}_{0}($ curl $) \times \boldsymbol{H}($ curl $)$, the mappings $t \mapsto\left(\left(\boldsymbol{E}^{(j)}, \boldsymbol{H}^{(j)}\right)(t),(\boldsymbol{v}, \boldsymbol{w})\right)_{\boldsymbol{X}}, j=1,2$ are absolutely continuous from $[0, T]$ to $\mathbb{R}$. By definition, the difference $(\boldsymbol{e}, \boldsymbol{h}):=\left(\boldsymbol{E}^{(1)}-\right.$ $\left.\boldsymbol{E}^{(2)}, \boldsymbol{H}^{(1)}-\boldsymbol{H}^{(2)}\right)$ satisfies $(\boldsymbol{e}, \boldsymbol{h})(0)=0$ and

$$
\begin{array}{r}
\frac{d}{d t} \int_{\Omega} \epsilon \boldsymbol{e}(t) \cdot \boldsymbol{v}+\mu \boldsymbol{h}(t) \cdot \boldsymbol{w} d x+\int_{\Omega} \boldsymbol{e}(t) \cdot \operatorname{curl} \boldsymbol{w}-\boldsymbol{h}(t) \cdot \operatorname{curl} \boldsymbol{v} d x \\
=\int_{\Omega} \epsilon\left(\hat{\boldsymbol{E}}^{(2)}(t)-\hat{\boldsymbol{E}}^{(1)}(t)\right) \cdot \boldsymbol{v}+\mu\left(\hat{\boldsymbol{H}}^{(2)}(t)-\hat{\boldsymbol{H}}^{(1)}(t)\right) \cdot \boldsymbol{w} d x \\
\text { for a.e. in }(0, T) \text { and all }(\boldsymbol{v}, \boldsymbol{w}) \in \boldsymbol{H}_{0}(\operatorname{curl}) \times \boldsymbol{H}(\operatorname{curl}),
\end{array}
$$

for some $\left(\hat{\boldsymbol{E}}^{(j)}, \hat{\boldsymbol{H}}^{(j)}\right) \in L^{\infty}((0, T), \boldsymbol{X}), j=1,2$, satisfying

$$
\left(\hat{\boldsymbol{E}}^{(j)}, \hat{\boldsymbol{H}}^{(j)}\right)(t) \in \partial \varphi\left(\left(\boldsymbol{E}^{(j)}, \boldsymbol{H}^{(j)}\right)(t)\right) \text { for a.e. } t \in(0, T) .
$$


In view of (4.20), the classical result by Ball [3] implies that $(\boldsymbol{e}, \boldsymbol{h}) \in \mathcal{C}([0, T], \boldsymbol{X})$ satisfies the variation by constants formula

$$
(\boldsymbol{e}, \boldsymbol{h})(t)=\int_{0}^{t} \mathbb{T}_{t-s}\left(\left(\hat{\boldsymbol{E}}^{(2)}-\hat{\boldsymbol{E}}^{(1)}, \hat{\boldsymbol{H}}^{(2)}-\hat{\boldsymbol{H}}^{(1)}\right)(s)\right) d s, \quad \forall t \in[0, T]
$$

Therefore, we obtain from the energy balance equality (Lemma 2.1) together with $(\boldsymbol{e}, \boldsymbol{h})(0)=0$ that

$$
\begin{aligned}
& \|(\boldsymbol{e}, \boldsymbol{h})(t)\|_{\boldsymbol{X}}^{2}=2 \int_{0}^{t}\left(\left(\hat{\boldsymbol{E}}^{(2)}-\hat{\boldsymbol{E}}^{(1)}, \hat{\boldsymbol{H}}^{(2)}-\hat{\boldsymbol{H}}^{(1)}\right)(s),(\boldsymbol{e}, \boldsymbol{h})(s)\right)_{\boldsymbol{X}} d s \\
& =-2 \int_{0}^{t}\left(\left(\hat{\boldsymbol{E}}^{(2)}-\hat{\boldsymbol{E}}^{(1)}, \hat{\boldsymbol{H}}^{(2)}-\hat{\boldsymbol{H}}^{(1)}\right)(s),\left(\boldsymbol{E}^{(2)}-\boldsymbol{E}^{(1)}, \boldsymbol{H}^{(2)}-\boldsymbol{H}^{(1)}\right)(s)\right)_{\boldsymbol{X}} d s \leq 0,
\end{aligned}
$$

for all $t \in[0, T]$, where we have used the monotonicity property of the subdifferential $\partial \varphi$ to get the above inequality. In conclusion, $\left(\boldsymbol{E}^{(1)}, \boldsymbol{H}^{(1)}\right)=\left(\boldsymbol{E}^{(2)}, \boldsymbol{H}^{(2)}\right)$. Then, applying $(\boldsymbol{e}, \boldsymbol{h})=0$ to $(4.20)$, we obtain for a.e. $t \in(0, T)$ and all $(\boldsymbol{v}, \boldsymbol{w}) \in \boldsymbol{H}_{0}(\mathbf{c u r l}) \times$ $\boldsymbol{H}(\mathbf{c u r l})$ that

$$
\int_{\Omega} \epsilon\left(\hat{\boldsymbol{E}}^{(2)}(t)-\hat{\boldsymbol{E}}^{(1)}(t)\right) \cdot \boldsymbol{v}+\mu\left(\hat{\boldsymbol{H}}^{(2)}(t)-\hat{\boldsymbol{H}}^{(1)}(t)\right) \cdot \boldsymbol{w} d x=0
$$

from which it follows that $\left(\hat{\boldsymbol{E}}^{(1)}, \hat{\boldsymbol{H}}^{(1)}\right)=\left(\hat{\boldsymbol{E}}^{(2)}, \hat{\boldsymbol{H}}^{(2)}\right)$.

4.3. Final Step: $(3.2) \Rightarrow(3.3)$. Let $(\mathbf{f}, \mathbf{g}) \in L^{1}((0, T), \boldsymbol{X})$ and $\left(\boldsymbol{E}_{0}, \boldsymbol{H}_{0}\right) \in \boldsymbol{X}$. Further, let $(\boldsymbol{E}, \boldsymbol{H}) \in \mathcal{C}([0, T], \boldsymbol{X})$ denote the unique solution to (3.2), as we have already proved in the previous steps. In other words, for every $(\boldsymbol{v}, \boldsymbol{w}) \in \boldsymbol{H}_{0}(\mathbf{c u r l}) \times$ $\boldsymbol{H}(\mathbf{c u r l})$, the mapping $t \mapsto((\boldsymbol{E}, \boldsymbol{H})(t),(\boldsymbol{v}, \boldsymbol{w}))_{\boldsymbol{X}}$ is absolutely continuous from $[0, T]$ to $\mathbb{R}$, and it holds that

$$
\left\{\begin{array}{l}
\frac{d}{d t} \int_{\Omega} \epsilon \boldsymbol{E}(t) \cdot \boldsymbol{v}+\mu \boldsymbol{H}(t) \cdot \boldsymbol{w} d x+\int_{\Omega} \boldsymbol{E}(t) \cdot \operatorname{curl} \boldsymbol{w}-\boldsymbol{H}(t) \cdot \operatorname{curl} \boldsymbol{v} d x \\
+\int_{\Omega} \epsilon \hat{\boldsymbol{E}}(t) \cdot \boldsymbol{v}+\mu \hat{\boldsymbol{H}}(t) \cdot \boldsymbol{w} d x=\int_{\Omega} \mathbf{f}(t) \cdot \boldsymbol{v}+\mathbf{g}(t) \cdot \boldsymbol{w} d x \\
\text { for a.e. } t \in(0, T) \text { and all }(\boldsymbol{v}, \boldsymbol{w}) \in \boldsymbol{H}_{0}(\operatorname{curl}) \times \boldsymbol{H}(\mathbf{c u r l}) \\
(\boldsymbol{E}, \boldsymbol{H})(0)=\left(\boldsymbol{E}_{0}, \boldsymbol{H}_{0}\right)
\end{array}\right.
$$

with a unique $(\hat{\boldsymbol{E}}, \hat{\boldsymbol{H}}) \in L^{\infty}((0, T), \boldsymbol{X})$ satisfying $(\hat{\boldsymbol{E}}, \hat{\boldsymbol{H}})(t) \in \partial \varphi((\boldsymbol{E}, \boldsymbol{H})(t))$ for a.e. $t \in(0, T)$. Using again the classical result by Ball [3], the variational formulation (4.22) is equivalent to the variation of constants formula:

$$
\begin{array}{r}
(\boldsymbol{E}, \boldsymbol{H})(t)=\mathbb{T}_{t}\left(\boldsymbol{E}_{0}, \boldsymbol{H}_{0}\right)+\int_{0}^{t} \mathbb{T}_{t-s}\left(\left(\epsilon^{-1} \mathbf{f}, \mu^{-1} \mathbf{g}\right)(s)-(\hat{\boldsymbol{E}}, \hat{\boldsymbol{H}})(t)\right) d s \\
\forall t \in[0, T] .
\end{array}
$$

Then, applying the energy balance equality (Lemma 2.1) to (4.23), we obtain that

$$
\begin{array}{r}
\|(\boldsymbol{E}, \boldsymbol{H})(t)\|_{\boldsymbol{X}}^{2}=\left\|\left(\boldsymbol{E}_{0}, \boldsymbol{H}_{0}\right)\right\|_{\boldsymbol{X}}^{2}+2 \int_{0}^{t}\left(\left(\epsilon^{-1} \mathbf{f}, \mu^{-1} \mathbf{g}\right)(t)-(\hat{\boldsymbol{E}}, \hat{\boldsymbol{H}})(t),(\boldsymbol{E}, \boldsymbol{H})(t)\right)_{\boldsymbol{X}} d t, \\
\forall t \in[0, T],
\end{array}
$$


from which it follows that the mapping $t \mapsto\|(\boldsymbol{E}, \boldsymbol{H})(t)\|_{\boldsymbol{X}}^{2}$ is absolutely continuous from $[0, T]$ to $\mathbb{R}$ with

$$
\frac{1}{2} \frac{d}{d t}\|(\boldsymbol{E}, \boldsymbol{H})(t)\|_{\boldsymbol{X}}^{2}=\left(\left(\epsilon^{-1} \mathbf{f}, \mu^{-1} \mathbf{g}\right)(t)-(\hat{\boldsymbol{E}}, \hat{\boldsymbol{H}})(t),(\boldsymbol{E}, \boldsymbol{H})(t)\right)_{\boldsymbol{X}}
$$

for a.e. $t \in(0, T)$.

Since $(\hat{\boldsymbol{E}}, \hat{\boldsymbol{H}})(t) \in \partial \varphi((\boldsymbol{E}, \boldsymbol{H})(t))$ holds for a.e. $t \in(0, T)$, we obtain from the definition of the subdifferential that

$$
\begin{array}{r}
-((\hat{\boldsymbol{E}}, \hat{\boldsymbol{H}})(t),(\boldsymbol{E}, \boldsymbol{H})(t))_{\boldsymbol{X}} \leq-((\hat{\boldsymbol{E}}, \hat{\boldsymbol{H}})(t),(\boldsymbol{v}, \boldsymbol{w}))_{\boldsymbol{X}}+\varphi(\boldsymbol{v}, \boldsymbol{w})-\varphi((\boldsymbol{E}, \boldsymbol{H})(t)), \\
\text { for a.e. } t \in(0, T) \text { and all }(\boldsymbol{v}, \boldsymbol{w}) \in \boldsymbol{X} .
\end{array}
$$

Then, applying (4.25) to (4.24) yields that

$$
\begin{aligned}
-\frac{d}{d t} \int_{\Omega} \epsilon \boldsymbol{E}(t) & \cdot \frac{1}{2} \boldsymbol{E}(t)+\mu \boldsymbol{H}(t) \cdot \frac{1}{2} \boldsymbol{H}(t) d x+\int_{\Omega} \mathbf{f}(t) \cdot \boldsymbol{E}(t)+\mathbf{g}(t) \cdot \boldsymbol{H}(t) d x \\
& -\int_{\Omega} \epsilon \hat{\boldsymbol{E}}(t) \cdot \boldsymbol{v}+\mu \hat{\boldsymbol{H}}(t) \cdot \boldsymbol{w} d x+\varphi(\boldsymbol{v}, \boldsymbol{w})-\varphi((\boldsymbol{E}, \boldsymbol{H})(t)) \geq 0,
\end{aligned}
$$

for a.e. $t \in(0, T)$ and all $(\boldsymbol{v}, \boldsymbol{w}) \in \boldsymbol{X}$.

Finally, adding (4.26) to the left hand side of the variational equality in (4.22), we see that $(\boldsymbol{E}, \boldsymbol{H})$ satisfies the Maxwell variational inequality (3.3).

5. Proof of Theorem 3.3. In the following, let $(\mathbf{f}, \mathbf{g}) \in \mathcal{C}^{0,1}([0, T], \boldsymbol{X})$ and $\left(\boldsymbol{E}_{0}, \boldsymbol{H}_{0}\right) \in \boldsymbol{H}_{0}($ curl $) \times \boldsymbol{H}($ curl $)$.

5.1. Uniqueness. Suppose that $\left(\boldsymbol{E}^{(j)}, \boldsymbol{H}^{(j)}\right) \in L^{\infty}\left((0, T), \boldsymbol{H}_{0}(\mathbf{c u r l}) \times \boldsymbol{H}(\mathbf{c u r l})\right) \cap$ $W^{1, \infty}((0, T), \boldsymbol{X}), j=1,2$, are solutions to $(\mathrm{VI})$. Setting the test function $(\boldsymbol{v}, \boldsymbol{w})=$ $\left(\boldsymbol{E}^{(2)}, \boldsymbol{H}^{(2)}\right)(t)$ in the variational inequality for $\left(\boldsymbol{E}^{(1)}, \boldsymbol{H}^{(1)}\right)$ and the test function $(\boldsymbol{v}, \boldsymbol{w})=\left(\boldsymbol{E}^{(1)}, \boldsymbol{H}^{(1)}\right)(t)$ in the variational inequality for $\left(\boldsymbol{E}^{(2)}, \boldsymbol{H}^{(2)}\right)$, and then adding the resulting inequalities, we obtain for the difference $(\boldsymbol{e}, \boldsymbol{h}):=\left(\boldsymbol{E}^{(1)}-\boldsymbol{E}^{(2)}, \boldsymbol{H}^{(1)}-\right.$ $\left.\boldsymbol{H}^{(2)}\right)$ that

$$
\begin{array}{r}
-\int_{\Omega} \epsilon \frac{d}{d t} \boldsymbol{e}(t) \cdot \boldsymbol{e}(t)+\mu \frac{d}{d t} \boldsymbol{h}(t) \cdot \boldsymbol{h}(t) d x-\int_{\Omega} \operatorname{curl} \boldsymbol{H}^{(1)}(t) \cdot \boldsymbol{E}^{(2)}(t) d x \\
+\int_{\Omega} \operatorname{curl} \boldsymbol{E}^{(1)}(t) \cdot \boldsymbol{H}^{(2)}(t) d x-\int_{\Omega} \operatorname{curl} \boldsymbol{H}^{(2)}(t) \cdot \boldsymbol{E}^{(1)}(t) d x \\
\quad+\int_{\Omega} \operatorname{curl} \boldsymbol{E}^{(2)}(t) \cdot \boldsymbol{H}^{(1)}(t) d x \geq 0, \quad \text { for a.e. } t \in(0, T) .
\end{array}
$$

On the other hand, we know that $\boldsymbol{E}^{(j)} \in \boldsymbol{H}_{0}(\mathbf{c u r l})$ and $\boldsymbol{H}^{(j)} \in \boldsymbol{H}(\mathbf{c u r l})$ for $j=1,2$ such that (2.1) implies

$$
\begin{array}{r}
-\int_{\Omega} \operatorname{curl} \boldsymbol{H}^{(1)}(t) \cdot \boldsymbol{E}^{(2)}(t) d x+\int_{\Omega} \operatorname{curl} \boldsymbol{E}^{(1)}(t) \cdot \boldsymbol{H}^{(2)}(t) d x \\
-\int_{\Omega} \operatorname{curl} \boldsymbol{H}^{(2)}(t) \cdot \boldsymbol{E}^{(1)}(t) d x+\int_{\Omega} \operatorname{curl} \boldsymbol{E}^{(2)}(t) \cdot \boldsymbol{H}^{(1)}(t) d x=0 .
\end{array}
$$

It follows therefore that

$$
0 \geq \int_{\Omega} \epsilon \frac{d}{d t} \boldsymbol{e}(t) \cdot \boldsymbol{e}(t)+\mu \frac{d}{d t} \boldsymbol{h}(t) \cdot \boldsymbol{h}(t) d x=\frac{1}{2} \frac{d}{d t}\|(\boldsymbol{e}, \boldsymbol{h})(t)\|_{\boldsymbol{X}}^{2} \quad \text { a.e. in }(0, T) .
$$

Integrating this inequality yields that $0 \geq\|(\boldsymbol{e}, \boldsymbol{h})(t)\|_{\boldsymbol{X}}^{2}-\|(\boldsymbol{e}, \boldsymbol{h})(0)\|_{\boldsymbol{X}}^{2}=\|(\boldsymbol{e}, \boldsymbol{h})(t)\|_{\boldsymbol{X}}^{2}$ for all $t \in[0, T]$. 
5.2. Existence. Let $\left\{\lambda_{n}\right\}_{n=1}^{\infty}$ be a null sequence of positive real numbers, and we consider again the integral equation (4.4). Thanks to the regularity $(\mathbf{f}, \mathbf{g}) \in$ $\mathcal{C}^{0,1}([0, T], \boldsymbol{X})$ and $\left(\boldsymbol{E}_{0}, \boldsymbol{H}_{0}\right) \in D(\mathcal{A})$, we may apply a well-known result from the semilinear semigroup theory (see [18, Theorem 1.6, p. 189] and [10, Corollary 7.6, p. $440])$ and obtain that (4.4) admits a unique solution $\left(\boldsymbol{E}_{n}, \boldsymbol{H}_{n}\right) \in \mathcal{C}([0, T], D(\mathcal{A})) \cap$ $\mathcal{C}^{1}([0, T], \boldsymbol{X})$ satisfying $\left(\boldsymbol{E}_{n}, \boldsymbol{H}_{n}\right)(0)=\left(\boldsymbol{E}_{0}, \boldsymbol{H}_{0}\right)$ and

$$
\frac{d}{d t}\left(\boldsymbol{E}_{n}, \boldsymbol{H}_{n}\right)(t)-\mathcal{A}\left(\boldsymbol{E}_{n}, \boldsymbol{H}_{n}\right)(t)=\left(\epsilon^{-1} \mathbf{f}, \mu^{-1} \mathbf{g}\right)(t)-\Phi_{\lambda_{n}}\left(\left(\boldsymbol{E}_{n}, \boldsymbol{H}_{n}\right)(t)\right), \quad \forall t \in[0, T] .
$$

Moreover, the sequence $\left\{\left(\boldsymbol{E}_{n}, \boldsymbol{H}_{n}\right)\right\}_{n=1}^{\infty}$ is bounded in $\mathcal{C}([0, T], D(\mathcal{A})) \cap \mathcal{C}^{1}([0, T], \boldsymbol{X})$. This boundedness result will be shown in Section 5.3. Therefore, as readily proven in Section 4.1 and due to the boundedness of $\left(\boldsymbol{E}_{n}, \boldsymbol{H}_{n}\right) \subset \mathcal{C}([0, T], D(\mathcal{A})) \cap \mathcal{C}^{1}([0, T], \boldsymbol{X})$, we can select a subsequence of $\left\{\lambda_{n}\right\}_{n=1}^{\infty}$ in (4.4), denoted again by the sequence itself, such that

$$
\begin{aligned}
\left(\boldsymbol{E}_{n}, \boldsymbol{H}_{n}\right) & \rightarrow(\boldsymbol{E}, \boldsymbol{H}) \quad \text { weakly star in } L^{\infty}((0, T), \boldsymbol{X}) \text { as } n \rightarrow \infty, \\
\Phi_{\lambda_{n}}\left(\boldsymbol{E}_{n}, \boldsymbol{H}_{n}\right) \rightarrow(\hat{\boldsymbol{E}}, \hat{\boldsymbol{H}}) & \text { weakly star in } L^{\infty}((0, T), \boldsymbol{X}) \text { as } n \rightarrow \infty,
\end{aligned}
$$

where $(\boldsymbol{E}, \boldsymbol{H}) \in L^{\infty}((0, T), D(\mathcal{A})) \cap W^{1, \infty}((0, T), \boldsymbol{X})$ and $(\hat{\boldsymbol{E}}, \hat{\boldsymbol{H}}) \in L^{\infty}((0, T), \boldsymbol{X})$ satisfy (3.2). Employing the higher regularity property $(\boldsymbol{E}, \boldsymbol{H}) \in L^{\infty}((0, T), D(\mathcal{A})) \cap$ $W^{1, \infty}((0, T), \boldsymbol{X})$ in $(3.2)$ and $\overline{D(\mathcal{A})}=\boldsymbol{X}$, we obtain that

$$
\left\{\begin{array}{l}
\int_{\Omega} \epsilon \frac{d}{d t} \boldsymbol{E}(t) \cdot \boldsymbol{v}+\mu \frac{d}{d t} \boldsymbol{H}(t) \cdot \boldsymbol{w} d x+\int_{\Omega} \operatorname{curl} \boldsymbol{E}(t) \cdot \boldsymbol{w}-\operatorname{curl} \boldsymbol{H}(t) \cdot \boldsymbol{v} d x \\
+\int_{\Omega} \epsilon \hat{\boldsymbol{E}}(t) \cdot \boldsymbol{v}+\mu \hat{\boldsymbol{H}}(t) \cdot \boldsymbol{w} d x=\int_{\Omega} \mathbf{f}(t) \cdot \boldsymbol{v}+\mathbf{g}(t) \cdot \boldsymbol{w} d x \\
\text { for a.e. } t \in(0, T) \text { and all }(\boldsymbol{v}, \boldsymbol{w}) \in \boldsymbol{X} \\
(\hat{\boldsymbol{E}}(t), \hat{\boldsymbol{H}}(t)) \in \partial \varphi((\boldsymbol{E}, \boldsymbol{H})(t)) \text { for a.e. } t \in(0, T), \\
(\boldsymbol{E}, \boldsymbol{H})(0)=\left(\boldsymbol{E}_{0}, \boldsymbol{H}_{0}\right) .
\end{array}\right.
$$

On the other hand, as readily proven in (4.26), it holds for a.e. $t \in(0, T)$ and all $(\boldsymbol{v}, \boldsymbol{w}) \in \boldsymbol{X}$ that

$$
\begin{aligned}
-\frac{d}{d t} \int_{\Omega} \epsilon \boldsymbol{E}(t) & \cdot \frac{1}{2} \boldsymbol{E}(t)+\mu \boldsymbol{H}(t) \cdot \frac{1}{2} \boldsymbol{H}(t) d x+\int_{\Omega} \mathbf{f}(t) \cdot \boldsymbol{E}(t)+\mathbf{g}(t) \cdot \boldsymbol{H}(t) d x \\
- & \int_{\Omega} \epsilon \hat{\boldsymbol{E}}(t) \cdot \boldsymbol{v}+\mu \hat{\boldsymbol{H}}(t) \cdot \boldsymbol{w} d x+\varphi(\boldsymbol{v}, \boldsymbol{w})-\varphi((\boldsymbol{E}, \boldsymbol{H})(t)) \geq 0,
\end{aligned}
$$

from which together with the regularity property $(\boldsymbol{E}, \boldsymbol{H}) \in W^{1, \infty}((0, T), \boldsymbol{X})$ it follows that

$$
\begin{array}{r}
-\int_{\Omega} \epsilon \frac{d}{d t} \boldsymbol{E}(t) \cdot \boldsymbol{E}(t)+\mu \frac{d}{d t} \boldsymbol{H}(t) \cdot \boldsymbol{H}(t) d x+\int_{\Omega} \mathbf{f}(t) \cdot \boldsymbol{E}(t)+\mathbf{g}(t) \cdot \boldsymbol{H}(t) d x \\
-\int_{\Omega} \epsilon \hat{\boldsymbol{E}}(t) \cdot \boldsymbol{v}+\mu \hat{\boldsymbol{H}}(t) \cdot \boldsymbol{w} d x+\varphi(\boldsymbol{v}, \boldsymbol{w})-\varphi((\boldsymbol{E}, \boldsymbol{H})(t)) \geq 0 .
\end{array}
$$

Adding (5.3) to the left hand side of the variational equality in (5.2), we conclude that $(\boldsymbol{E}, \boldsymbol{H})$ satisfies $(\mathrm{VI})$. 
5.3. Boundedness Result for (4.4). Let $t \in(0, T)$ and $n \in \mathbb{N}$. According to (4.4), for every $h \in(0, T-t)$, it holds that

$$
\begin{aligned}
\left(\boldsymbol{E}_{n}, \boldsymbol{H}_{n}\right)(t+h) \\
=\mathbb{T}_{t+h}\left(\boldsymbol{E}_{0}, \boldsymbol{H}_{0}\right)+\int_{0}^{t+h} \mathbb{T}_{t+h-s}\left(\left(\epsilon^{-1} \mathbf{f}, \mu^{-1} \mathbf{g}\right)(s)-\Phi_{\lambda_{n}}\left(\left(\boldsymbol{E}_{n}, \boldsymbol{H}_{n}\right)(s)\right)\right) d s \\
=\mathbb{T}_{t+h}\left(\boldsymbol{E}_{0}, \boldsymbol{H}_{0}\right)+\int_{0}^{h} \mathbb{T}_{t+h-s}\left(\left(\epsilon^{-1} \mathbf{f}, \mu^{-1} \mathbf{g}\right)(s)-\Phi_{\lambda_{n}}\left(\left(\boldsymbol{E}_{n}, \boldsymbol{H}_{n}\right)(s)\right)\right) d s \\
\quad+\int_{h}^{t+h} \mathbb{T}_{t+h-s}\left(\left(\epsilon^{-1} \mathbf{f}, \mu^{-1} \mathbf{g}\right)(s)-\Phi_{\lambda_{n}}\left(\left(\boldsymbol{E}_{n}, \boldsymbol{H}_{n}\right)(s)\right)\right) d s, \\
=\mathbb{T}_{t}\left(\mathbb{T}_{h}\left(\boldsymbol{E}_{0}, \boldsymbol{H}_{0}\right)+\int_{0}^{h} \mathbb{T}_{h-s}\left(\left(\epsilon^{-1} \mathbf{f}, \mu^{-1} \mathbf{g}\right)(s)-\Phi_{\lambda_{n}}\left(\left(\boldsymbol{E}_{n}, \boldsymbol{H}_{n}\right)(s)\right)\right) d s\right) \\
\quad+\int_{0}^{t} \mathbb{T}_{t-s}\left(\left(\epsilon^{-1} \mathbf{f}, \mu^{-1} \mathbf{g}\right)(s+h)-\Phi_{\lambda_{n}}\left(\left(\boldsymbol{E}_{n}, \boldsymbol{H}_{n}\right)(s+h)\right)\right) d s .
\end{aligned}
$$

Subtracting (4.4) from the above expression, it follows that

$$
\begin{aligned}
& \frac{\left(\boldsymbol{E}_{n}, \boldsymbol{H}_{n}\right)(t+h)-\left(\boldsymbol{E}_{n}, \boldsymbol{H}_{n}\right)(t)}{h} \\
& =\mathbb{T}_{t}\left(\frac{\mathbb{T}_{h}\left(\boldsymbol{E}_{0}, \boldsymbol{H}_{0}\right)-\left(\boldsymbol{E}_{0}, \boldsymbol{H}_{0}\right)}{h}+\frac{1}{h} \int_{0}^{h} \mathbb{T}_{h-s}\left(\left(\epsilon^{-1} \mathbf{f}, \mu^{-1} \mathbf{g}\right)(s)\right.\right. \\
& \left.\left.-\Phi_{\lambda_{n}}\left(\left(\boldsymbol{E}_{n}, \boldsymbol{H}_{n}\right)(s)\right)\right) d s\right)+\frac{1}{h} \int_{0}^{t} \mathbb{T}_{t-s}\left(\left(\epsilon^{-1} \mathbf{f}, \mu^{-1} \mathbf{g}\right)(s+h)-\left(\epsilon^{-1} \mathbf{f}, \mu^{-1} \mathbf{g}\right)(s)\right. \\
& \left.-\Phi_{\lambda_{n}}\left(\left(\boldsymbol{E}_{n}, \boldsymbol{H}_{n}\right)(s+h)\right)+\Phi_{\lambda_{n}}\left(\left(\boldsymbol{E}_{n}, \boldsymbol{H}_{n}\right)(s)\right)\right) d s .
\end{aligned}
$$

Then, applying the energy balance equality (Lemma 2.1) to the above variation of constants formula implies

$$
\begin{aligned}
& \left\|\frac{\left(\boldsymbol{E}_{n}, \boldsymbol{H}_{n}\right)(t+h)-\left(\boldsymbol{E}_{n}, \boldsymbol{H}_{n}\right)(t)}{h}\right\|_{\boldsymbol{X}}^{2} \\
& =\left\|\frac{\mathbb{T}_{h}\left(\boldsymbol{E}_{0}, \boldsymbol{H}_{0}\right)-\left(\boldsymbol{E}_{0}, \boldsymbol{H}_{0}\right)}{h}+\frac{1}{h} \int_{0}^{h} \mathbb{T}_{h-s}\left(\left(\epsilon^{-1} \mathbf{f}, \mu^{-1} \mathbf{g}\right)(s)-\Phi_{\lambda_{n}}\left(\left(\boldsymbol{E}_{n}, \boldsymbol{H}_{n}\right)(s)\right)\right) d s\right\|_{\boldsymbol{X}}^{2} \\
& +2 \int_{0}^{t}\left[\left(\frac{\left(\epsilon^{-1} \mathbf{f}, \mu^{-1} \mathbf{g}\right)(s+h)-\left(\epsilon^{-1} \mathbf{f}, \mu^{-1} \mathbf{g}\right)(s)}{h}, \frac{\left(\boldsymbol{E}_{n}, \boldsymbol{H}_{n}\right)(s+h)-\left(\boldsymbol{E}_{n}, \boldsymbol{H}_{n}\right)(s)}{h}\right)_{\boldsymbol{X}}\right. \\
& \left.-\frac{1}{h^{2}}\left(\Phi_{\lambda_{n}}\left(\left(\boldsymbol{E}_{n}, \boldsymbol{H}_{n}\right)(s+h)\right)-\Phi_{\lambda_{n}}\left(\left(\boldsymbol{E}_{n}, \boldsymbol{H}_{n}\right)(s)\right),\left(\boldsymbol{E}_{n}, \boldsymbol{H}_{n}\right)(s+h)-\left(\boldsymbol{E}_{n}, \boldsymbol{H}_{n}\right)(s)\right)_{\boldsymbol{X}}\right] d s \\
& =: I_{n}(h)+I I_{n}(h) .
\end{aligned}
$$

Since $\mathbb{T}_{h-s}: \boldsymbol{X} \rightarrow \boldsymbol{X}$ is unitary, the first term $I_{n}(h)$ can be estimated as follows:

$$
\begin{gathered}
I_{n}(h) \leq\left[\left\|\frac{\mathbb{T}_{h}\left(\boldsymbol{E}_{0}, \boldsymbol{H}_{0}\right)-\left(\boldsymbol{E}_{0}, \boldsymbol{H}_{0}\right)}{h}\right\|_{\boldsymbol{X}}+\frac{1}{h} \int_{0}^{h}\left\|\left(\epsilon^{-1} \mathbf{f}, \mu^{-1} \mathbf{g}\right)(s)-\Phi_{\lambda_{n}}\left(\left(\boldsymbol{E}_{n}, \boldsymbol{H}_{n}\right)(s)\right)\right\|_{\boldsymbol{X}} d s\right]^{2} \\
\leq\left[\left\|\frac{\mathbb{T}_{h}\left(\boldsymbol{E}_{0}, \boldsymbol{H}_{0}\right)-\left(\boldsymbol{E}_{0}, \boldsymbol{H}_{0}\right)}{h}\right\|_{\boldsymbol{X}}+\left\|\left(\epsilon^{-1} \mathbf{f}, \mu^{-1} \mathbf{g}\right)\right\|_{\mathcal{C}([0, T], \boldsymbol{X})}+\left\|\Phi_{\lambda_{n}}\left(\boldsymbol{E}_{n}, \boldsymbol{H}_{n}\right)\right\|_{\mathcal{C}([0, T], \boldsymbol{X})}\right]^{2} .
\end{gathered}
$$

Therefore, since $\left\{\Phi_{\lambda_{n}}\left(\boldsymbol{E}_{n}, \boldsymbol{H}_{n}\right)\right\}_{n=1}^{\infty} \subset \mathcal{C}([0, T], \boldsymbol{X})$ is bounded (see Section 4.1) and $\left(\boldsymbol{E}_{0}, \boldsymbol{H}_{0}\right) \in D(\mathcal{A})$, there exists a constant $c>0$, independent of $n, t$ and $h$, such that

$$
I_{n}(h) \leq c, \quad \forall h \in(0, T-t) .
$$


On the other hand, thanks to the monotonicity property of the Yosida approximation, the second term $I I_{n}(h)$ can be estimated as follows:

$$
\begin{aligned}
& I I_{n}(h) \\
& \leq 2 \int_{0}^{t}\left(\frac{\left(\epsilon^{-1} \mathbf{f}, \mu^{-1} \mathbf{g}\right)(s+h)-\left(\epsilon^{-1} \mathbf{f}, \mu^{-1} \mathbf{g}\right)(s)}{h}, \frac{\left(\boldsymbol{E}_{n}, \boldsymbol{H}_{n}\right)(s+h)-\left(\boldsymbol{E}_{n}, \boldsymbol{H}_{n}\right)(s)}{h}\right)_{\boldsymbol{X}} d s \\
& \leq 2 T\left\|\left(\epsilon^{-1} \mathbf{f}, \mu^{-1} \mathbf{g}\right)\right\|_{\mathcal{C}^{0,1}([0, T], \boldsymbol{X})}\left\|\frac{d}{d t}\left(\boldsymbol{E}_{n}, \boldsymbol{H}_{n}\right)\right\|_{\mathcal{C}([0, T], \boldsymbol{X})} \\
& \leq 2 T^{2}\left\|\left(\epsilon^{-1} \mathbf{f}, \mu^{-1} \mathbf{g}\right)\right\|_{\mathcal{C}^{0,1}([0, T], \boldsymbol{X})}^{2}+\frac{1}{2}\left\|\frac{d}{d t}\left(\boldsymbol{E}_{n}, \boldsymbol{H}_{n}\right)\right\|_{\mathcal{C}([0, T], \boldsymbol{X})}^{2}, \forall h \in(0, T-t) .
\end{aligned}
$$

In conclusion, it holds for all $h \in(0, T-t)$ that

$$
\begin{array}{r}
\left\|\frac{\left(\boldsymbol{E}_{n}, \boldsymbol{H}_{n}\right)(t+h)-\left(\boldsymbol{E}_{n}, \boldsymbol{H}_{n}\right)(t)}{h}\right\|_{\boldsymbol{X}}^{2} \leq c+2 T^{2}\left\|\left(\epsilon^{-1} \mathbf{f}, \mu^{-1} \mathbf{g}\right)\right\|_{\mathcal{C}^{0,1}([0, T], \boldsymbol{X})}^{2} \\
+\frac{1}{2}\left\|\frac{d}{d t}\left(\boldsymbol{E}_{n}, \boldsymbol{H}_{n}\right)\right\|_{\mathcal{C}([0, T], \boldsymbol{X})}^{2}
\end{array}
$$

Then, passing to the limit $h \rightarrow 0$,

$$
\left\|\frac{d}{d t}\left(\boldsymbol{E}_{n}, \boldsymbol{H}_{n}\right)(t)\right\|_{\boldsymbol{X}}^{2} \leq c+2 T^{2}\left\|\left(\epsilon^{-1} \mathbf{f}, \mu^{-1} \mathbf{g}\right)\right\|_{\mathcal{C}^{0,1}([0, T], \boldsymbol{X})}^{2}+\frac{1}{2}\left\|\frac{d}{d t}\left(\boldsymbol{E}_{n}, \boldsymbol{H}_{n}\right)\right\|_{\mathcal{C}([0, T], \boldsymbol{X})}^{2} .
$$

As $t \in(0, T)$ and $n \in \mathbb{N}$ were chosen arbitrarily, it follows therefore that

$$
\frac{1}{2}\left\|\frac{d}{d t}\left(\boldsymbol{E}_{n}, \boldsymbol{H}_{n}\right)\right\|_{\mathcal{C}([0, T], \boldsymbol{X})}^{2} \leq c+2 T^{2}\left\|\left(\epsilon^{-1} \mathbf{f}, \mu^{-1} \mathbf{g}\right)\right\|_{\mathcal{C}^{0,1}([0, T], \boldsymbol{X})}^{2}, \quad \forall n \in \mathbb{N}
$$

and consequently $\left\{\frac{d}{d t}\left(\boldsymbol{E}_{n}, \boldsymbol{H}_{n}\right)\right\}_{n=1}^{\infty} \subset \mathcal{C}([0, T], \boldsymbol{X})$ is bounded. Thus, from (5.1) and the boundedness of $\left\{\Phi_{\lambda_{n}}\left(\boldsymbol{E}_{n}, \boldsymbol{H}_{n}\right)\right\}_{n=1}^{\infty} \subset \mathcal{C}([0, T], \boldsymbol{X})$, we obtain that $\left\{\left(\boldsymbol{E}_{n}, \boldsymbol{H}_{n}\right)\right\}_{n=1}^{\infty}$ is bounded in $\mathcal{C}([0, T], D(\mathcal{A})) \cap \mathcal{C}^{1}([0, T], \boldsymbol{X})$.

\section{REFERENCES}

[1] J.-P. Aubin and H. Frankowska. Set-valued analysis. Modern Birkhäuser Classics. Birkhäuser Boston, Inc., Boston, MA, 2009.

[2] A. Azevedo, F. Miranda, and L. Santos. Variational and quasivariational inequalities with first order constraints. J. Math. Anal. Appl., 397(2):738-756, 2013.

[3] J.M. Ball. Strongly continuous semigroups, weak solutions, and the variation of constants formula. Proc. Amer. Math. Soc., 64:370-373, 1977.

[4] J. W. Barrett and L. Prigozhin. Bean's critical-state model as the $p \rightarrow \infty$ limit of an evolutionary p-Laplacian equation. Nonlinear Anal., 42(6):977-993, 2000.

[5] J. W. Barrett and L. Prigozhin. Dual formulations in critical state problems. Interfaces Free Bound., 8(3):349-370, 2006.

[6] J. W. Barrett and L. Prigozhin. Existence and approximation of a mixed formulation for thin film magnetization problems in superconductivity. Math. Models Methods Appl. Sci., 24(5):991-1015, 2014.

[7] J. W. Barrett and L. Prigozhin. Sandpiles and superconductors: nonconforming linear finite element approximations for mixed formulations of quasi-variational inequalities. IMA J. Numer. Anal., 35(1):1-38, 2015.

[8] C. P. Bean. Magnetization of high-field superconductors. Rev. Mod. Phys., 36:31-39, 1964. 
[9] G. Duvaut and J.-L. Lions. Inequalities in mechanics and physics. Springer-Verlag, Berlin-New York, 1976. Translated from the French by C. W. John, Grundlehren der Mathematischen Wissenschaften, 219.

[10] K.-J. Engel and R. Nagel. One-parameter semigroups for linear evolution equations, volume 194 of Graduate Texts in Mathematics. Springer-Verlag, New York, 2000. With contributions by S. Brendle, M. Campiti, T. Hahn, G. Metafune, G. Nickel, D. Pallara, C. Perazzoli, A. Rhandi, S. Romanelli and R. Schnaubelt.

[11] F. Jochmann. On a first-order hyperbolic system including Bean's model for superconductors with displacement current. J. Differential Equations, 246(6):2151-2191, 2009.

[12] F. Jochmann. Well-posedness for Bean's critical state model with displacement current. J. Math. Anal. Appl., 362(2):505-513, 2010.

[13] R. Leis. Initial-boundary value problems in mathematical physics. B. G. Teubner, Stuttgart, 1986.

[14] A. Milani. On a variational inequality with time dependent convex constraints for the Maxwell equations. Rend. Sem. Mat. Univ. Politec. Torino, 36:389-401, 1979.

[15] A. Milani. On a variational inequality with time dependent convex constraint for the Maxwell equations. II. Rend. Sem. Mat. Univ. Politec. Torino, 43(1):171-183, 1985.

[16] F. Miranda, J.-F. Rodrigues, and L. Santos. A class of stationary nonlinear Maxwell systems. Math. Models Methods Appl. Sci., 19(10):1883-1905, 2009.

[17] F. Miranda, J.-F. Rodrigues, and L. Santos. On a $p$-curl system arising in electromagnetism. Discrete Contin. Dyn. Syst. Ser. S, 5(3):605-629, 2012.

[18] A. Pazy. Semigroups of Linear Operators and Applications to Partial Differential Equations. Springer-Verlag, New York, 1983.

[19] L. Prigozhin. The Bean model in superconductivity: variational formulation and numerical solution. J. Comput. Phys., 129(1):190-200, 1996.

[20] L. Prigozhin. On the Bean critical-state model in superconductivity. European J. Appl. Math., $7(3): 237-247,1996$.

[21] L. Prigozhin. Variational inequalities in critical-state problems. Phys. D, 197(3-4):197-210, 2004.

[22] R. E. Showalter. Monotone operators in Banach space and nonlinear partial differential equations, volume 49 of Mathematical Surveys and Monographs. American Mathematical Society, Providence, RI, 1997.

[23] I. Yousept. Optimal Control of Quasilinear $\boldsymbol{H}$ (curl)-Elliptic Partial Differential Equations in Magnetostatic Field Problems. SIAM J. Control Optim., 51(5):3624-3651, 2013.

[24] I. Yousept. Hyperbolic Maxwell variational inequalities for Bean's critical-state model in type-II superconductivity. SIAM J. Numer. Anal., 55(5):2444-2464, 2017.

[25] I. Yousept. Optimal control of non-smooth hyperbolic evolution Maxwell equations in type-II superconductivity. SIAM J. Control Optim., 55(4):2305-2332, 2017. 